\title{
Groups with finitely many conjugacy classes and their automorphisms
}

\author{
Ashot Minasyan*
}

\begin{abstract}
We combine classical methods of combinatorial group theory with the theory of small cancellation over relatively hyperbolic groups to construct finitely generated torsion-free groups that have only finitely many classes of conjugate elements. Moreover, we present several results concerning embeddings into such groups.

As another application of these techniques, we prove that every countable group $C$ can be realized as a group of outer automorphisms of a group $N$, where $N$ is a finitely generated group having Kazhdan's property ( $\mathrm{T}$ ) and containing exactly two conjugacy classes.
\end{abstract}

Mathematics Subject Classification (2000). 20F65, 20E45, 20 F28.

Keywords. Conjugacy classes, relatively hyperbolic groups, outer automorphism groups.

\section{Introduction}

We shall start with the following definition.

Definition. Suppose that $n \geq 2$ is an integer. We will say that a group $M$ has the property $(n \mathrm{CC})$ if there are exactly $n$ conjugacy classes of elements in $M$.

Note that a group $M$ has (2CC) if and only if any two non-trivial elements are conjugate in $M$. For two elements $x, y$ of some group $G$, we shall write $x \stackrel{G}{\sim} y$ if $x$ and $y$ are conjugate in $G$, and $x \underset{\sim}{\sim} y$ if they are not.

For a group $G$, denote by $\pi(G)$ the set of all finite orders of elements of $G$. A classical theorem of G. Higman, B. H. Neumann and H. Neumann ([8]) states that every countable group $G$ can be embedded into a countable (but infinitely generated) group $M$, where any two elements of the same order are conjugate and $\pi(M)=\pi(G)$.

For any integer $n \geq 2$, take $G=\mathbb{Z} / 2^{n-2} \mathbb{Z}$ and embed $G$ into a countable group $M$ according to the theorem above. Then $\operatorname{card}(\pi(M))=\operatorname{card}(\pi(G))=n-1$. Since, in addition, $M$ will always contain an element of infinite order, the theorem of Higman-Neumann-Neumann implies that $G$ has $(n C C)$.

\footnotetext{
${ }^{*}$ This work was supported by the Swiss National Science Foundation Grant \# PP002-68627.
} 
Another way to construct infinite groups with finitely many conjugacy classes was suggested by S. Ivanov [15, Theorem 41.2], who showed that for every sufficiently large prime $p$ there is an infinite 2-generated group $M_{p}$ of exponent $p$ possessing exactly $p$ conjugacy classes. The group $M_{p}$ is constructed as a direct limit of word hyperbolic groups, and, as noted in [21], it is impossible to obtain an infinite group with $(2 \mathrm{CC})$ in the same manner.

In the recent paper [21] D. Osin developed a theory of small cancellation over relatively hyperbolic groups and used it to obtain the following remarkable result:

Theorem 1.1 ([21], Theorem 1.1). Any countable group $G$ can be embedded into a 2-generated group $M$ such that any two elements of the same order are conjugate in $M$ and $\pi(M)=\pi(G)$.

Applying this theorem to the group $G=\mathbb{Z} / 2^{n-2} \mathbb{Z}$ one can show that for each integer $n \geq 2$ there exists a 2 -generated group with $(n \mathrm{CC})$. And when $n=2$ we get a 2-generated torsion-free group that has exactly two conjugacy classes.

The presence of elements of finite orders in the above constructions was important, because if two elements have different orders, they can never be conjugate. So, naturally, one can ask the following

Question 1. Do there exist torsion-free (finitely generated) groups with $(n \mathrm{CC})$, for any integer $n \geq 3$ ?

Note that if $G$ is the finitely generated group with (2CC) constructed by Osin, then the $m$-th direct power $G^{m}$ of $G$ is also a finitely generated torsion-free group which satisfies $\left(2^{m} \mathrm{CC}\right)$. But what if we want to achieve a torsion-free group with (3CC)? With this purpose one could come up with

Question 2. Suppose that $G$ is a countable torsion-free group and $x, y \in G$ are non-conjugate. Is it possible to embed $G$ into a group $M$, which has (3CC), so that $x$ and $y$ stay non-conjugate in $M$ ?

Unfortunately, the answer to Question 2 is negative as the following example shows.

Example 1. Consider the group

$$
G_{1}=\left\langle a, t \mid t a t^{-1}=a^{-1}\right\rangle
$$

which is isomorphic to the non-trivial semidirect product $\mathbb{Z} \rtimes \mathbb{Z}$. Note that $G_{1}$ is torsion-free, and $t$ is not conjugated to $t^{-1}$ in $G_{1}$ because $t \nsim t^{-1}$ in the infinite cyclic group $\langle t\rangle$ which is canonically isomorphic to the quotient of $G_{1}$ by the normal closure of $a$. However, if $G_{1}$ is embedded into a (3CC)-group $M$, it is easy to see 
that every element of $M$ will be conjugated to its inverse (indeed, if $y \in M \backslash\{1\}$ and $y \stackrel{M}{\sim} y^{-1}$ then $y^{\epsilon} \stackrel{M}{\sim} a \stackrel{M}{\sim} a^{-1}$, for some $\epsilon \in\{1,-1\}$, hence $y^{\epsilon} \stackrel{M}{\sim} y^{-\epsilon}-\mathrm{a}$ contradiction). In particular, $t \stackrel{M}{\sim} t^{-1}$.

An analog of the above example can be given for each $n \geq 3-$ see Section 3. This example shows that, in order to get a positive result, one would have to strengthen the assumptions of Question 2.

Let $G$ be a group. Two elements $x, y \in G$ are said to be commensurable if there exist $k, l \in \mathbb{Z} \backslash\{0\}$ such that $x^{k}$ is conjugate to $y^{l}$. We will use the notation $x \stackrel{G}{\approx} y$ if $x$ and $y$ are commensurable in $G$. In the case when $x$ is not commensurable with $y$ we will write $x \stackrel{G}{\not} \not$ y. Observe that commensurability, as well as conjugacy, defines an equivalence relation on the set of elements of $G$. It is somewhat surprising that if one replaces the words "non-conjugate" with the words "non-commensurable" in Question 2, the answer becomes positive:

Corollary 1.2. Assume that $G$ is a countable torsion-free group, $n \in \mathbb{N}, n \geq 2$, and $x_{1}, \ldots, x_{n-1} \in G \backslash\{1\}$ are pairwise non-commensurable. Then there exists a group $M$ and an injective homomorphism $\varphi: G \rightarrow M$ such that

1. $M$ is torsion-free and generated by two elements;

2. $M$ has $(n \mathrm{CC})$;

3. $M$ is 2-boundedly simple;

4. the elements $\varphi\left(x_{1}\right), \ldots, \varphi\left(x_{n-1}\right)$ are pairwise non-commensurable in $M$.

Recall that a group $G$ is said to be $k$-boundedly simple if for any $x, y \in G \backslash$ $\{1\}$ there exist $l \leq k, \epsilon_{1}, \ldots, \epsilon_{l} \in\{-1,1\}$ and $g_{1}, \ldots, g_{l} \in G$ such that $x=$ $g_{1} y^{\epsilon_{1}} g_{1}^{-1} \ldots g_{l} y^{\epsilon_{l}} g_{l}^{-1}$ in $G$.

A group is called boundedly simple if it is $k$-boundedly simple for some $k \in \mathbb{N}$. Evidently every boundedly simple group is simple; the converse is not true in general. For example, the infinite alternating group $A_{\infty}$ is simple but not boundedly simple because conjugation preserves the type of the decomposition of a permutation into a product of cycles. First examples of torsion-free finitely generated boundedly simple groups were constructed by A. Muranov (see [12, Theorem 2], [13, Theorem 1]).

Corollary 1.2 is an immediate consequence of a more general Theorem 3.5 that will be proved in Section 3.

Applying Corollary 1.2 to the group $G=F\left(x_{1}, \ldots, x_{n-1}\right)$, which is free on the set $\left\{x_{1}, \ldots, x_{n-1}\right\}$, and its non-commensurable elements $x_{1}, \ldots, x_{n-1}$, we obtain a positive answer to Question 1:

Corollary 1.3. For every integer $n \geq 3$ there exists a torsion-free 2-boundedly simple group satisfying $(n \mathrm{CC})$ and generated by two elements. 
(In the case when $n=2$ the above statement was obtained by Osin in [21, Corollary 1.3].) In fact, for any (finitely generated) torsion-free group $H$ we can set $G=H * F\left(x_{1}, \ldots, x_{n-1}\right)$, and then use Corollary 1.2 to embed $G$ into a group $M$ enjoying the properties $1-4$ from its claim. Since there is a continuum of pairwise non-isomorphic 2-generated torsion-free groups ([4]), and a finitely generated group can contain at most countably many of different 2-generated subgroups, this shows that there must be continually many pairwise non-isomorphic groups satisfying properties 1-3 from Corollary 1.2.

Recall that the rank of a group $G, \operatorname{rank}(G)$, is the minimal number of elements required to generate $G$. In Section 4 we show how classical theory of HNN-extensions allows to construct different embeddings into (infinitely generated) groups that have finitely many classes of conjugate elements, and in Section 5 we use Osin's results (from [21]) regarding quotients of relatively hyperbolic groups to prove

Theorem 1.4. Let $H$ be a torsion-free countable group and let $M \triangleleft H$ be a nontrivial normal subgroup. Then $H$ can be isomorphically embedded into a torsion-free group $Q$, possessing a normal subgroup $N \triangleleft Q$, such that

- $Q=H \cdot N$ and $H \cap N=M$ (hence $Q / N \cong H / M$ );

- $N$ has (2CC);

- for all $x, y \in Q \backslash\{1\}, x \stackrel{Q}{\sim} y$ if and only if $\varphi(x) \stackrel{Q / N}{\sim} \varphi(y)$, where $\varphi: Q \rightarrow Q / N$ is the natural homomorphism;

- $\operatorname{rank}(N)=2$ and $\operatorname{rank}(Q) \leq \operatorname{rank}(H / M)+2$.

This theorem implies that if $Q / N \cong H / M$ has exactly $(n-1)$ conjugacy classes (e.g., if it is finite), then the group $Q$ will have ( $n \mathrm{CC}$ ) and will not be simple (if $n \geq 3$ ). Thus it may be used to build ( $n \mathrm{CC}$ )-groups in a recursive manner. It also allows to obtain embeddings of countable torsion-free groups into $(n C C)$-groups, which we could not get by using Corollary 1.2. For instance, as we saw in Example 1, the fundamental group of the Klein bottle $G_{1}$, given by (1.1), can not be embedded into a (3CC)-group $M$ so that $t \stackrel{M}{x} t^{-1}$. However, with 4 conjugacy classes this is already possible: see Corollary 5.5 in Section 5. The idea is as follows: the group $G_{1}$ can be mapped onto $\mathbb{Z} / 3 \mathbb{Z}$ in such a way that the images of the elements $t$ and $t^{-1}$ are distinct. Let $M$ be the kernel of this homomorphism. One can apply Theorem 1.4 to the pair $\left(G_{1}, M\right)$ to obtain the required embedding of $G_{1}$ into a group $Q$. And since $\mathbb{Z} / 3 \mathbb{Z}$ has exactly 3 conjugacy classes, the group $Q$ will have (4CC).

An application of Theorem 1.4 to the case when $H=\mathbb{Z}$ and $M=2 \mathbb{Z} \triangleleft H$ also provides an affirmative answer to a question of A. Izosov from [9, Question 11.42], asking whether there exists a torsion-free (3CC)-group $Q$ that contains a normal subgroup $N$ of index 2 . 
The goal of the second part of this article is to show that every countable group can be realized as a group of outer automorphisms of some finitely generated (2CC)group. This problem has some historical background: in [11] T. Matumoto proved that every group is a group of outer automorphisms of some group (in contrast, there are groups, e.g., $\mathbb{Z}$, that are not full automorphism groups of any group); M. Droste, M. Giraudet, R. Göbel ([7]) showed that for every group $C$ there exists a simple group $S$ such that $\operatorname{Out}(S) \cong C$; I. Bumagina and D. Wise in [3] proved that each countable group $C$ is isomorphic to $\operatorname{Out}(N)$ where $N$ is a 2-generated subgroup of a countable $C^{\prime}(1 / 6)$-group, and if, in addition, $C$ is finitely presented then one can choose $N$ to be residually finite.

In Section 6 we establish a few useful statements regarding paths in the Cayley graph of a relatively hyperbolic group $G$, and apply them in Section 7 to obtain small cancellation quotients of $G$ satisfying certain conditions. Finally, in Section 8 we prove the following

Theorem 1.5. Let $C$ be an arbitrary countable group. Then for every non-elementary torsion-free word hyperbolic group $F_{1}$ there exists a torsion-free group $N$ satisfying the following properties:

- $N$ is a 2-generated quotient of $F_{1}$;

- $N$ has (2CC);

- $\operatorname{Out}(N) \cong C$.

The principal difference between this theorem and the result of [3] is that our group $N$ is torsion-free and simple. Moreover, if one applies Theorem 1.5 to the case when $F_{1}$ is a torsion-free hyperbolic group with Kazhdan's property (T) (and recalls that every quotient of a group with property $(\mathrm{T})$ also has $(\mathrm{T})$ ), one will get

Corollary 1.6. For any countable group $C$ there is a 2-generated group $N$ such that $N$ has (2CC) and Kazhdan's property (T), and $\operatorname{Out}(N) \cong C$.

The reason why Kazhdan's property $(\mathrm{T})$ is interesting in this context is the question from [6, p. 134] which asked whether there exist groups that satisfy property (T) and have infinite outer automorphism groups (it can be motivated by a theorem of F. Paulin [22] which claims that the outer automorphism group is finite for any word hyperbolic group with property (T)). Positive answers to this question were obtained (using different methods) by Y. Ollivier and D. Wise [14], Y. de Cornulier [5], and I. Belegradek and D. Osin [2]. Corollary 1.6 not only shows that the group of outer automorphisms of a group $N$ with property (T) can be infinite, but also demonstrates that there are no restrictions whatsoever on $\operatorname{Out}(N)$.

Acknowledgements. The author would like to thank D. Osin for fruitful discussions and encouragement. 


\section{Relatively hyperbolic groups}

Assume that $G$ is a group, $\left\{H_{\lambda}\right\}_{\lambda \in \Lambda}$ is a fixed collection of subgroups of $G$ (called peripheral subgroups), and $\mathcal{X}$ is a subset of $G$. The subset $\mathcal{X}$ is called a relative generating set of $G$ with respect to $\left\{H_{\lambda}\right\}_{\lambda \in \Lambda}$ if $G$ is generated by $\mathcal{X} \cup \cup_{\lambda \in \Lambda} H_{\lambda}$. In this case $G$ a quotient of the free product

$$
F=\left(*_{\lambda \in \Lambda} H_{\lambda}\right) * F(\mathcal{X})
$$

where $F(\mathcal{X})$ is the free group with basis $\mathcal{X}$. Let $\mathcal{R}$ be a subset of $F$ such that the kernel of the natural epimorphism $F \rightarrow G$ is the normal closure of $\mathcal{R}$ in the group $F$; then we will say that $G$ has relative presentation

$$
\left\langle\mathcal{X},\left\{H_{\lambda}\right\}_{\lambda \in \Lambda} \mid R=1, R \in \mathcal{R}\right\rangle \text {. }
$$

If the sets $\mathcal{X}$ and $\mathcal{R}$ are finite, the relative presentation (2.1) is said to be finite.

Set $\mathscr{H}=\bigsqcup_{\lambda \in \Lambda}\left(H_{\lambda} \backslash\{1\}\right)$. A finite relative presentation (2.1) is said to satisfy a linear relative isoperimetric inequality if there exists $C>0$ such that, for every word $w$ in the alphabet $\mathcal{X} \cup \mathscr{H}$ (for convenience, we will further assume that $\mathcal{X}^{-1}=\mathcal{X}$ ) representing the identity in the group $G$, one has

$$
w \stackrel{F}{=} \prod_{i=1}^{k} f_{i}^{-1} R_{i}^{ \pm 1} f_{i},
$$

with equality in the group $F$, where $R_{i} \in \mathcal{R}, f_{i} \in F$, for $i=1, \ldots, k$, and $k \leq C\|w\|$, where $\|w\|$ is the length of the word $w$.

The next definition is due to Osin (see [20]):

Definition. The group $G$ is called hyperbolic relative to (the collection of peripheral subgroups) $\left\{H_{\lambda}\right\}_{\lambda \in \Lambda}$, if $G$ admits a finite relative presentation (2.1) satisfying a linear relative isoperimetric inequality.

This definition is independent of the choice of the finite generating set $\mathcal{X}$ and the finite set $\mathcal{R}$ in (2.1) (see [20]). We would also like to note that, in general, it does not require the group $G$ to be finitely generated, which will be important in this paper. The definition immediately implies the following basic facts:

Remark 2.1 ([20]). (a) Let $\left\{H_{\lambda}\right\}_{\lambda \in \Lambda}$ be an arbitrary family of groups. Then the free product $G=*_{\lambda \in \Lambda} H_{\lambda}$ will be hyperbolic relative to $\left\{H_{\lambda}\right\}_{\lambda \in \Lambda}$.

(b) Any word hyperbolic group (in the sense of Gromov) is hyperbolic relative to the family $\{\{1\}\}$, where $\{1\}$ denotes the trivial subgroup. 
Recall that a group $H$ is called elementary if it has a cyclic subgroup of finite index. Further in this section we will assume that $G$ is a non-elementary group hyperbolic relative to a family of proper subgroups $\left\{H_{\lambda}\right\}_{\lambda \in \Lambda}$.

An element $g \in G$ is said to be parabolic if it is conjugated to an element of $H_{\lambda}$ for some $\lambda \in \Lambda$. Otherwise $g$ is said to be hyperbolic. Given a subgroup $S \leq G$, we denote by $S^{0}$ the set of all hyperbolic elements of $S$ of infinite order.

Lemma 2.2 ([17], Theorem 4.3, Corollary 1.7). For every $g \in G^{0}$ the following conditions hold.

1) The element $g$ is contained in a unique maximal elementary subgroup $E_{G}(g)$ of $G$, where

$$
E_{G}(g)=\left\{f \in G \mid f g^{n} f^{-1}=g^{ \pm n} \text { for some } n \in \mathbb{N}\right\} .
$$

2) The group $G$ is hyperbolic relative to the collection $\left\{H_{\lambda}\right\}_{\lambda \in \Lambda} \cup\left\{E_{G}(g)\right\}$.

Recall that a non-trivial subgroup $H \leq G$ is called malnormal if for every $g \in$ $G \backslash H, H \cap g g^{-1}=\{1\}$. The next lemma is a special case of Theorem 1.4 from [20]:

Lemma 2.3. For any $\lambda \in \Lambda$ and any $g \notin H_{\lambda}$, the intersection $H_{\lambda} \cap g H_{\lambda} g^{-1}$ is finite. If $h \in G, \mu \in \Lambda$ and $\mu \neq \lambda$, then the intersection $H_{\lambda} \cap h H_{\mu} h^{-1}$ is finite. In particular, if $G$ is torsion-free then $H_{\lambda}$ is malnormal (provided that $H_{\lambda} \neq\{1\}$ ).

Lemma 2.4 ([20], Theorem 2.40). Suppose that a group $G$ is hyperbolic relative to a collection of subgroups $\left\{H_{\lambda}\right\}_{\lambda \in \Lambda} \cup\left\{S_{1}, \ldots, S_{m}\right\}$, where $S_{1}, \ldots, S_{m}$ are word hyperbolic (in the ordinary non-relative sense). Then $G$ is hyperbolic relative to $\left\{H_{\lambda}\right\}_{\lambda \in \Lambda}$.

Lemma 2.5 ([19], Corollary 1.4). Let $G$ be a group which is hyperbolic relative to a collection of subgroups $\left\{H_{\lambda}\right\}_{\lambda \in \Lambda} \cup\{K\}$. Suppose that $K$ is finitely generated and there is a monomorphism $\alpha: K \rightarrow H_{v}$ for some $v \in \Lambda$. Then the HNN-extension $\left\langle G, t \mid t x t^{-1}=\alpha(x), x \in K\right\rangle$ is hyperbolic with respect to $\left\{H_{\lambda}\right\}_{\lambda \in \Lambda}$.

In [21] Osin introduced the following notion: a subgroup $S \leq G$ is suitable if there exist two elements $g_{1}, g_{2} \in S^{0}$ such that $g_{1} \not \approx g_{2}$ and $E_{G}\left(g_{1}\right) \cap E_{G}\left(g_{2}\right)=\{1\}$.

For any $S \leq G$ with $S^{0} \neq \emptyset$, one sets

$$
E_{G}(S)=\bigcap_{g \in S^{0}} E_{G}(g)
$$

which is obviously a subgroup of $G$ normalized by $S$. Note that $E_{G}(S)=\{1\}$ if the subgroup $S$ is suitable in $G$. As shown in [1, Lemma 3.3], if $S$ is non-elementary and $S^{0} \neq \emptyset$ then $E_{G}(S)$ is the unique maximal finite subgroup of $G$ normalized by $S$. 
Lemma 2.6. Let $\{H\}_{\lambda \in \Lambda}$ be a family of groups and let $F$ be a torsion-free nonelementary word hyperbolic group. Then the free product $G=\left(* *_{\lambda \in \Lambda} H_{\lambda}\right) * F$ is hyperbolic relative to $\left\{H_{\lambda}\right\}_{\lambda \in \Lambda}$ and $F$ is a suitable subgroup of $G$.

Proof. Indeed, $G$ is hyperbolic relative to $\left\{H_{\lambda}\right\}_{\lambda \in \Lambda}$ by Remark 2.1 and Lemma 2.4. Since $F$ is non-elementary, there are elements of infinite order $x, y \in F$ such that $F$

$x \not \approx y$ (see, for example, [16, Lemma 3.2]). Evidently, $x$ and $y$ are hyperbolic elements of $G$ that are not commensurable with each other, and the subgroups $E_{G}(x)=E_{F}(x) \leq F, E_{G}(y)=E_{F}(y) \leq F$ are cyclic (as elementary subgroups of a torsion-free group). Hence $E_{G}(x) \cap E_{G}(y)=\{1\}$, and thus $F$ is suitable in $G$.

Lemma 2.7 ([21], Lemma 2.3). Suppose that $G$ is a group hyperbolic relative to a family of subgroups $\left\{H_{\lambda}\right\}_{\lambda \in \Lambda}$ and $S \leq G$ is a suitable subgroup. Then one can find infinitely many pairwise non-commensurable (in $G$ ) elements $g_{1}, g_{2}, \ldots \in S^{0}$ such that $E_{G}\left(g_{i}\right) \cap E_{G}\left(g_{j}\right)=\{1\}$ for all $i \neq j$.

The following theorem was proved by Osin in [21] using the theory of small cancellation over relatively hyperbolic groups, and represents our main tool for obtaining new quotients of such groups having a number of prescribed properties:

Theorem 2.8 ([21], Theorem 2.4). Let $G$ be a torsion-free group hyperbolic relative to a collection of subgroups $\left\{H_{\lambda}\right\}_{\lambda \in \Lambda}$, let $S$ be a suitable subgroup of $G$, and let $T$, $U$ be arbitrary finite subsets of $G$. Then there exist a group $G_{1}$ and an epimorphism $\eta: G \rightarrow G_{1}$ such that:

(i) The restriction of $\eta$ to $\bigcup_{\lambda \in \Lambda} H_{\lambda} \cup U$ is injective, and the group $G_{1}$ is hyperbolic relative to the collection $\left\{\eta\left(H_{\lambda}\right)\right\}_{\lambda \in \Lambda}$;

(ii) for every $t \in T$, we have $\eta(t) \in \eta(S)$;

(iii) $\eta(S)$ is a suitable subgroup of $G_{1}$;

(iv) $G_{1}$ is torsion-free;

(v) the kernel $\operatorname{ker}(\eta)$ of $\eta$ is generated (as a normal subgroup of $G$ ) by a finite collection of elements belonging to $T \cdot S$.

We have slightly changed the original formulation of the above theorem from [21], demanding the injectivity on $V=\bigcup_{\lambda \in \Lambda} H_{\lambda} \cup U$ (instead of just $\bigcup_{\lambda \in \Lambda} H_{\lambda}$ ) and adding the last statement concerning the generators of the kernel. The latter follows from the explicit form of the relations, imposed on $G$ (see the proof of Theorem 2.4 in [21]), and the former from part 2 of Lemma 5.1 in [21] and the fact that any element from $V$ has length (in the alphabet $\mathcal{X} \cup \mathscr{H}$ ) at most $N$, where $N=\max \left\{|h|_{X \cup \mathscr{H}} \mid h \in U\right\}+1$. 


\section{Groups with finitely many conjugacy classes}

Lemma 3.1. Let $G$ be a group and let $x_{1}, x_{2}, x_{3}, x_{4} \in G$ be elements of infinite order G

such that $x_{1} \not \approx x_{i}, i=2,3,4$. Let $H=\left\langle G, t \mid t x_{3} t^{-1}=x_{4}\right\rangle$ be the HNN-extension of $G$ with associated cyclic subgroups generated by $x_{3}$ and $x_{4}$. Then $\begin{array}{rl}H & H \\ x_{1} & \approx x_{2} .\end{array}$

Proof. Arguing by contradiction, assume that $h x_{1}^{l} h^{-1} x_{2}^{m}=1$ for some $h \in H$, $l, m \in \mathbb{Z} \backslash\{0\}$. The element $h$ has a reduced presentation of the form

$$
h=g_{0} t^{\epsilon_{1}} g_{1} t^{\epsilon_{2}} \ldots t^{\epsilon_{k}} g_{k}
$$

where $g_{0}, \ldots, g_{k} \in G, \epsilon_{1}, \ldots, \epsilon_{k} \in \mathbb{Z} \backslash\{0\}$, and

$$
\begin{cases}g_{j} \notin\left\langle x_{3}\right\rangle & \text { if } 1 \leq j \leq k-1 \text { and } \epsilon_{j}>0, \epsilon_{j+1}<0, \\ g_{j} \notin\left\langle x_{4}\right\rangle & \text { if } 1 \leq j \leq k-1 \text { and } \epsilon_{j}<0, \epsilon_{j+1}>0 .\end{cases}
$$

By the assumptions, $x_{1} \not \approx x_{2}$ hence $k \geq 1$, and in the group $H$ we have

$$
h x_{1}^{l} h^{-1} x_{2}^{m}=g_{0} t^{\epsilon_{1}} g_{1} t^{\epsilon_{2}} \ldots t^{\epsilon_{k}} g_{k} x_{1}^{l} g_{k}^{-1} t^{-\epsilon_{k}} \ldots t^{-\epsilon_{2}} g_{1}^{-1} t^{-\epsilon_{1}} \tilde{g}_{0}=1,
$$

where $\tilde{g}_{0}=g_{0}^{-1} x_{2}^{m} \in G$. By Britton's Lemma (see [10, IV.2]), the left-hand side in (3.1) can not be reduced, and this can happen only if $g_{k} x_{1}^{l} g_{k}^{-1}$ belongs to either $\left\langle x_{3}\right\rangle$ or $\left\langle x_{4}\right\rangle$ in $G$, which would contradict the assumptions. Thus the lemma is proved.

Definition. Suppose that $G$ is a group and $X_{i} \subset G, i \in I$, is a family of subsets. We shall say that $X_{i}, i \in I$, are independent if no element of $X_{i}$ is commensurable with an element of $X_{j}$ whenever $i \neq j, i, j \in I$.

Lemma 3.2. Assume that $G$ is a countable torsion-free group, $n \in \mathbb{N}, n \geq 2$, and non-empty subsets $X_{i} \subset G \backslash\{1\}, i=1, \ldots, n-1$, are independent in $G$. Then $G$ can be (isomorphically) embedded into a countable torsion-free group $M$ in such a way that $M$ has $(n \mathrm{CC})$ and the subsets $X_{i}, i=1, \ldots, n-1$, remain independent in $M$.

Proof. For each $i=1, \ldots, n-1$, fix an element $x_{i} \in X_{i}$. First we embed $G$ into a countable torsion-free group $G_{1}$ such that for each non-trivial element $g \in G$ there exist $j \in\{1, \ldots, n-1\}$ and $t \in G_{1}$ satisfying $\operatorname{tg} t^{-1}=x_{j}$ in $G_{1}$, and the subsets $X_{i}, i=1, \ldots, n-1$, stay independent in $G_{1}$.

Let $g_{1}, g_{2}, \ldots$ be an enumeration of all non-trivial elements of $G$. Set $G(0)=G$ and suppose that we have already constructed the group $G(k)$, containing $G$, so that for each $l \in\{1, \ldots, k\}$ there is $j \in\{1, \ldots, n-1\}$ such that the element $g_{l}$ is conjugated in $G(k)$ to $x_{j}$, and $X_{i}, i=1, \ldots, n-1$, are independent in $G(k)$. 
Suppose, at first, that $g_{k+1}$ is commensurable in $G(k)$ with an element of $X_{j}$ for

some $j$. Then $g_{k+1} \stackrel{G(k)}{\not} h$ for every $h \in \bigcup_{i=1, i \neq j}^{n-1} X_{i}$. Define $G(k+1)$ to be the HNN-extension $\left\langle G(k), t_{k+1} \mid t_{k+1} g_{k+1} t_{k+1}^{-1}=x_{j}\right\rangle$. By Lemma 3.1 the subsets $X_{i}$, $i=1, \ldots, n-1$, will remain independent in $G(k+1)$.

Thus we can assume that $g_{k+1}$ is not commensurable with any element from $\bigcup_{i=1}^{n-1} X_{i}$ in $G(k)$. According to the induction hypotheses one can apply Lemma 3.1 to the HNN-extension

$$
G(k+1)=\left\langle G(k), t_{k+1} \mid t_{k+1} g_{k+1} t_{k+1}^{-1}=x_{1}\right\rangle
$$

to see that the subsets $X_{i} \subset G \leq G(k+1), i=1, \ldots, n-1$, are independent in $G(k+1)$.

Now, set $G_{1}=\bigcup_{k=0}^{\infty} G(k)$. Evidently $G_{1}$ has the required properties. In the same manner, one can embed $G_{1}$ into a countable torsion-free group $G_{2}$ so that each non-trivial element of $G_{1}$ will be conjugated to $x_{i}$ in $G_{2}$, for some $i \in\{1, \ldots, n-1\}$, and the subsets $X_{i}, i=1, \ldots, n-1$, continue to be independent in $G_{2}$.

Proceeding like that we obtain the desired group $M=\bigcup_{s=1}^{\infty} G_{s}$. By the construction, $M$ is a torsion-free countable group which has exactly $n$ conjugacy classes: [1], $\left[x_{1}\right], \ldots,\left[x_{n-1}\right]$. The subsets $X_{i}, i=1, \ldots, n-1$, are independent in $M$ because they are independent in $G_{s}$ for each $s \in \mathbb{N}$.

Corollary 3.3. In Lemma 3.2 one can add that the group $M$ is 2-boundedly simple.

Proof. Let a torsion-free countable group $G$ and its non-empty independent subsets $X_{i}, i=1, \ldots, n-1$, be as in Lemma 3.2. Let $F=F\left(a_{1}, \ldots, a_{n-1}, b_{1}, \ldots, b_{n-1}\right)$ be the free group with the free generating set $\left\{a_{1}, \ldots, a_{n-1}, b_{1}, \ldots, b_{n-1}\right\}$, and consider the group $\bar{G}=G * F$. For each $i=1, \ldots, n-1$, define

$$
\bar{X}_{i}=X_{i} \cup\left\{a_{i}, a_{i}^{-1}\right\} \cup\left\{\left[a_{j}, b_{i}\right] \mid j=1, \ldots, n-1, j \neq i\right\} \subset \bar{G},
$$

where $\left[a_{j}, b_{i}\right]=a_{j} b_{i} a_{j}^{-1} b_{i}^{-1}$. Using the universal properties of free groups and free products one can easily see that the subsets $\bar{X}_{i}, i=1, \ldots, n-1$, are independent in $\bar{G}$.

Now we apply Lemma 3.2 to find a countable torsion-free ( $n$ CC)-group $M$, containing $\bar{G}$, such that $\bar{X}_{i}, i=1, \ldots, n-1$, are independent in $M$. Observe that this implies that for any given $i=1, \ldots, n-1$, any two elements of $\bar{X}_{i}$ are conjugate in $M$. For arbitrary $x, y \in M \backslash\{1\}$ there exist $i, j \in\{1, \ldots, n-1\}$ such that $x \stackrel{M}{\sim} a_{i}$ and $y \stackrel{M}{\sim} a_{j}$. If $i=j$ then $x \stackrel{M}{\sim} y$. Otherwise, $y \stackrel{M}{\sim} a_{j} \stackrel{M}{\sim} a_{j}^{-1}$ and $x \stackrel{M}{\sim}\left[a_{j}, b_{i}\right]$ which is a product of two conjugates of $a_{j}$, and, hence, of $y$. Therefore the group $M$ is 2-boundedly simple, and since $G \leq \bar{G} \leq M$, the corollary is proved. 
Below is a particular (torsion-free) case of a theorem proved by Osin ([21, Theorem 2.6]):

Lemma 3.4. Any countable torsion-free group $S$ can be embedded into a 2-generated group $M$ so that $S$ is malnormal in $M$ and every element of $M$ is conjugated to an element of $S$ in $M$.

Proof. Following Osin's proof of Theorem 2.6 from [21], we see that the required group $M$ can be constructed as an inductive limit of relatively hyperbolic groups $G(i), i \in \mathbb{N}$. More precisely, one sets $G(0)=S * F_{2}$, where $F_{2}$ is a free group of rank $2, \xi_{0}=\operatorname{id}_{G(0)}: G(0) \rightarrow G(0)$, and for each $i \in \mathbb{N}$ one constructs a group $G(i)$ and an epimorphism $\xi_{i}: G(0) \rightarrow G(i)$ so that $\xi_{i}$ is injective on $S, G(i)$ is torsionfree and hyperbolic relative to $\left\{\xi_{i}(S)\right\}$, and $\xi_{i}$ factors through $\xi_{i-1}$. The group $M$ is defined to be the direct limit of $\left(G(i), \xi_{i}\right)$ as $i \rightarrow \infty$, i.e., $Q=G(0) / N$ where $N=\bigcup_{i \in \mathbb{N}} \operatorname{ker}\left(\xi_{i}\right)$. By Lemma 2.3, $\xi_{i}(S)$ is malnormal in $G(i)$, hence the image of $S$ will also be malnormal in $M$.

Theorem 3.5. Let $G$ be a torsion-free countable group, $n \in \mathbb{N}, n \geq 2$, and nonempty subsets $X_{i} \subset G \backslash\{1\}, i=1, \ldots, n-1$, be independent in $G$. Then $G$ can be embedded into a 2-generated torsion-free group $M$ which has $(n \mathrm{CC})$, so that the subsets $X_{i}, i=1, \ldots, n-1$, stay independent in $M$. Moreover, one can choose $M$ to be 2-boundedly simple.

Proof. First, according to Corollary 3.3, we can embed the group $G$ into a countable torsion-free group $S$ such that $S$ has $(n \mathrm{CC})$ and is 2-boundedly simple, and $X_{i}$, $i=1, \ldots, n-1$, are independent in $S$. Second, we apply Lemma 3.4 to find the 2-generated group $M$ from its claim. Choose any $i, j \in\{1, \ldots, n-1\}, i \neq j$, and $x \in X_{i}, y \in X_{j}$. If $x$ and $y$ were commensurable in $M$, the malnormality of $S$ would imply that $x$ and $y$ must be commensurable in $S$, contradicting the construction. Hence $X_{i}, i=1, \ldots, n-1$, are independent in $M$. Since each element of $M$ is conjugated to an element of $S$, it is evident that $M$ has $(n \mathrm{CC})$, is torsion-free and 2-boundedly simple.

Remark 3.6. A more direct proof of Theorem 3.5, not using Lemma 3.4, can be extracted from the proof of Theorem 5.1 (see Section 5), applied to the case when $H=M$.

It is easy to see that Theorem 3.5 immediately implies Corollary 1.2 that was formulated in the Introduction. As promised, we now give a counterexample to Question 2 (formulated in the Introduction) for any $n \geq 3$.

Example 2. Let $G_{2}=\left\langle a, t \mid t a t^{-1}=a^{2}\right\rangle$ be the Baumslag-Solitar BS(1,2)-group. Then $G_{2}$ is torsion-free, and the elements $t^{2}, t^{4}, \ldots, t^{2^{n-1}}$ are pairwise non-conjugate 
in $G_{2}$ (since this holds in the quotient of $G_{2}$ by the normal closure of $a$ ). Suppose that $G_{2}$ is embedded into a group $M$ having $(n \mathrm{CC})$ so that $t^{2}, t^{4}, \ldots, t^{2^{n-1}}$ are pairwise non-conjugate in $M$. Then $t^{2}, \ldots, t^{2^{n-1}}$ is the list of representatives of all non-trivial conjugacy classes of $M$. Therefore there exist $k, l \in\{1, \ldots, n-1\}$ such that $t \stackrel{M}{\sim} t^{2^{k}}$ and $a \stackrel{M}{\sim} t^{2^{l}}$. Consequently

$$
t^{2} \stackrel{M}{\sim} t^{2^{k+1}} \text { and } t^{2^{l}} \stackrel{M}{\sim} a \stackrel{M}{\sim} a^{2} \stackrel{M}{\sim} t^{2^{l+1}},
$$

hence $k=l=n-1$ according to the assumptions. But this yields

$$
t \stackrel{M}{\sim} t^{2^{n-1}} \stackrel{M}{\sim} a \stackrel{M}{\sim} a^{2} \stackrel{M}{\sim} t^{2}
$$

implying that $t^{2} \stackrel{M}{\sim} t^{4}$, which contradicts our assumptions.

Thus $G_{2}$ can not be embedded into a $(n C C)$-group $M$ in such a way that the elements $t^{2}, \ldots, t^{2^{n-1}}$ remain pairwise non-conjugate in $M$.

\section{Normal subgroups with $(n \mathrm{CC})$}

If $M$ is a normal subgroup of a group $H$, then $H$ naturally acts on $M$ by conjugation. We shall say that this action preserves the conjugacy classes of $M$ if for any $h \in H$ and $a \in M$ there exists $b \in M$ such that $h a h^{-1}=b a b^{-1}$.

Lemma 4.1. Let $G$ be a torsion-free group, $N \triangleleft G$ and $x_{1}, \ldots, x_{l} \in N \backslash\{1\}$ be pairwise non-commensurable (in $G$ ) elements. Then there exists a partition $N \backslash\{1\}=$ $\bigsqcup_{k=1}^{l} X_{k}$ of $N \backslash\{1\}$ into a (disjoint) union of $G$-independent subsets $X_{1}, \ldots, X_{l}$ such that $x_{k} \in X_{k}$ for every $k \in\{1, \ldots, l\}$. Moreover, each subset $X_{k}$ will be invariant under conjugation by elements of $G$.

Proof. Since $\underset{\approx}{\approx}$ is an equivalence relation on $G \backslash\{1\}$, one can find the corresponding decomposition: $G \backslash\{1\}=\bigsqcup_{j \in J} Y_{j}$, where $Y_{j}$ is an equivalence class for each $j \in J$. For each $k=1, \ldots, l$, there exists $j(k) \in J$ such that $x_{k} \in Y_{j(k)}$. Note that $j(k) \neq j(m)$ if $k \neq m$ since $x_{k} \not{G} x_{m}$.

Denote $J^{\prime}=J \backslash\{j(1), \ldots, j(l-1)\}$,

$$
X_{1}=Y_{j(1)} \cap N, \ldots, X_{l-1}=Y_{j(l-1)} \cap N, \text { and } X_{l}=\bigcup_{j \in J^{\prime}} Y_{j} \cap N .
$$

Evidently $N \backslash\{1\}=\bigsqcup_{k=1}^{l} X_{k}, X_{1}, \ldots, X_{l}$ are independent subsets of $G$ and $x_{k} \in$ $X_{k}$ for each $k=1, \ldots, l$. The final property follows from the construction since for any $a \in G$ and $j \in J$ we have $a Y_{j} a^{-1}=Y_{j}$ and $a N a^{-1}=N$. 
Lemma 4.2. For every countable group $C$ and each $n \in \mathbb{N}, n \geq 2$, there exists a countable torsion-free group $H$ having a normal subgroup $M \triangleleft H$ such that

(i) M satisfies $(n \mathrm{CC})$;

(ii) $M$ is 2-boundedly simple;

(iii) the natural action of $H$ on $M$ preserves the conjugacy classes of $M$;

(iv) $H / M \cong C$.

Proof. Let $H_{0}^{\prime}$ be the free group of infinite countable rank. Choose $N_{0}^{\prime} \triangleleft H_{0}^{\prime}$ so that $H_{0}^{\prime} / N_{0}^{\prime} \cong C$. Let $F=F\left(x_{1}, \ldots, x_{n-1}\right)$ denote the free group freely generated by $x_{1}, \ldots, x_{n-1}$. Define $H_{0}=H_{0}^{\prime} * F$ and let $N_{0}$ be the normal closure of $N_{0}^{\prime} \cup F$ in $H_{0}$. Evidently, $H_{0} / N_{0} \cong H_{0}^{\prime} / N_{0}^{\prime} \cong C$ and the elements $x_{1}, \ldots, x_{n-1} \in N_{0} \backslash\{1\}$ are pairwise non-commensurable in $H_{0}$.

By Lemma 4.1, one can choose a partition of $N_{0} \backslash\{1\}$ into the union of $H_{0^{-}}$ independent subsets:

$$
N_{0} \backslash\{1\}=\bigsqcup_{k=1}^{n-1} X_{0 k},
$$

so that $x_{k} \in X_{0 k}$ for each $k=1, \ldots, n-1$.

By Corollary 3.3 there exists a countable torsion-free 2-boundedly simple group $M_{1}$ with the property $(n \mathrm{CC})$ containing a copy of $N_{0}$, such that the subsets $X_{0 k}$, $k=1,2, \ldots, n-1$, are independent in $M_{1}$. Denote by $H_{1}=H_{0} * N_{0} M_{1}$ the amalgamated product of $H_{0}$ and $M_{1}$ along $N_{0}$, and let $N_{1}$ be the normal closure of $M_{1}$ in $H_{1}$. Note that $H_{1}$ is torsion-free as an amalgamated product of two torsion-free groups ([10, IV.2.7]).

We need to verify that the elements $x_{1}, \ldots, x_{n-1}$ are pairwise non-commensurable in $H_{1}$. Indeed, if $a \in X_{0 k}$ and $b \in X_{0 l}, k \neq l$, are conjugate in $H_{1}$ then there must exist $y_{1}, \ldots, y_{t} \in M_{1} \backslash N_{0}$ and $z_{1}, \ldots, z_{t-1} \in H_{0} \backslash N_{0}, z_{0}, z_{t} \in H_{0}$ such that

$$
z_{0} y_{1} \ldots z_{t-1} y_{t} z_{t} a z_{t}^{-1} y_{t}^{-1} z_{t-1}^{-1} \ldots y_{1}^{-1} z_{0}^{-1} \stackrel{H_{1}}{=} b .
$$

Suppose that $t$ is minimal possible with this property. As conjugation by elements of $H_{0}$ preserves $X_{0 k}$ and $X_{0 l}$, we can assume that $z_{0}, z_{t}=1$. Hence

$$
y_{1} z_{1} \ldots z_{t-1} y_{t} a y_{t}^{-1} z_{t-1}^{-1} \ldots z_{1}^{-1} y_{1}^{-1} b^{-1} \stackrel{H_{1}}{=} 1 .
$$

By the properties of amalgamated products (see [10, Chapter IV]), the left-hand side in this equality can not be reduced, consequently $y_{t} a y_{t}^{-1} \in N_{0} \backslash\{1\}=\bigsqcup_{k=1}^{n-1} X_{0 k}$. But then $y_{t} a y_{t}^{-1} \in X_{0 k}$ by the properties of $M_{1}$, contradicting the minimality of $t$. Thus, we have shown that $x_{k} \not H_{1} x_{l}$ whenever $k \neq l$. 
Assume that the group $H_{i}=H_{i-1} *_{N_{i-1}} M_{i}, i \geq 1$, has already been constructed, so that

0) $H_{i}$ is countable and torsion-free;

1) $N_{i-1} \triangleleft H_{i-1}$;

2) $H_{i-1}=H_{0} \cdot N_{i-1}$ and $H_{0} \cap N_{i-1}=N_{0}$;

3) $M_{i}$ satisfies $(n \mathrm{CC})$;

4) $x_{1}, \ldots, x_{n-1}$ are pairwise non-commensurable in $H_{i}$.

Let $N_{i}$ be the normal closure of $M_{i}$ in $H_{i}$. Because of the condition 4) and Lemma 4.1, one can find a partition of $N_{i} \backslash\{1\}$ into a union of $H_{i}$-independent subsets:

$$
N_{i} \backslash\{1\}=\bigsqcup_{k=1}^{n-1} X_{i k},
$$

so that $x_{k} \in X_{i k}$ for each $k=1, \ldots, n-1$. By Lemma 3.2 there is a countable group a $M_{i+1}$, with $(n \mathrm{CC})$, containing a copy of $N_{i}$, in which the subsets $X_{i k}$, $i=1, \ldots, n-1$, remain independent. Set $H_{i+1}=H_{i} *_{N_{i}} M_{i+1}$. Now, it is easy to verify that the analogs of the conditions 0 )-3) hold for $H_{i+1}$ and

$$
N_{i-1} \leq M_{i} \leq N_{i} \leq M_{i+1} .
$$

The analog of the condition 4) is true in $H_{i+1}$ by the same considerations as before (in the case of $H_{1}$ ).

Define the group $H=\bigcup_{i=1}^{\infty} H_{i}$ and its subgroup $M=\bigcup_{i=1}^{\infty} N_{i}$. Observe that the condition 0) implies that $H$ is torsion-free, condition 1) implies that $M$ is normal in $H$, and 2) implies that $H=H_{0} \cdot M$ and $H_{0} \cap M=N_{0}$. Hence $H / M \cong H_{0} /\left(H_{0} \cap M\right) \cong C$. Applying (4.1) we get $M=\bigcup_{i=1}^{\infty} M_{i}$, and thus, by the conditions 3$), 4)$ it enjoys the property $(n \mathrm{CC})$ : each element of $M$ will be a conjugate of $x_{k}$ for some $k \in\{1, \ldots, n-1\}$. Since $x_{1}, \ldots, x_{n-1} \in M_{1} \leq M$ and $M_{1}$ is 2-boundedly simple, then so will be $M$. Finally, 4) implies that $x_{k} \underset{H}{\sim} x_{l}$ whenever $k \neq l$, and, consequently, the natural action of $H$ on $M$ preserves its conjugacy classes.

Lemma 4.3. Suppose that $G$ is a group, $N \triangleleft G, A, B \leq G$ and $\varphi: A \rightarrow B$ is an isomorphism such that $\varphi(a) \in a N$ (i.e., the canonical images of a and $\varphi(a)$ in $G / N$ coincide) for each $a \in A$. Let $L=\langle G, t|$ tat $^{-1}=\varphi(a)$ for all $\left.a \in A\right\rangle$ be the $H N N$-extension of $G$ with associated subgroups $A$ and $B$, and let $K$ be the normal closure of $\langle N, t\rangle$ in $L$. Then $G \cap K=N$.

Proof. This statement easily follows from the universal property of HNN-extensions and is left as an exercise for the reader. 
The next lemma will allow us to construct $(n \mathrm{CC})$-groups that are not simple:

Lemma 4.4. Assume that $H$ is a torsion-free countable group and $M \triangleleft H$ is a nontrivial normal subgroup. Then $H$ can be isomorphically embedded into a countable torsion-free group $G$ possessing a normal subgroup $K \triangleleft G$ such that

1) $G=H K$ and $H \cap K=M$;

2) for all $x, y \in G \backslash\{1\}, \varphi(x)=\varphi(y)$ if and only if there exists an $h \in K$ such that $x=h y h^{-1}$, where $\varphi: G \rightarrow G / K$ is the natural homomorphism; in particular, $K$ will have (2CC);

3) $x \stackrel{G}{\sim} y$ if and only if $\varphi(x) \stackrel{G / K}{\sim} \varphi(y)$ for all $x, y \in G \backslash\{1\}$;

Proof. Choose a set of representatives $Z \subset H$ of cosets of $H$ modulo $M$, in such a way that each coset is represented by a unique element from $Z$ and $1 \notin Z$.

Define $G(0)=H$ and $K(0)=M$. Enumerate the elements of $G(0) \backslash\{1\}$ : $g_{1}, g_{2}, \ldots$. First we embed the group $G(0)$ into a countable torsion-free group $G_{1}$, having a normal subgroup $K_{1} \triangleleft G_{1}$, such that $G_{1}=H K_{1}, H \cap K_{1}=M$ and for every $i \geq 0$ there are $t_{i} \in K_{1}$ and $z_{i} \in Z$ satisfying $t_{i} g_{i} t_{i}^{-1}=z_{i}$.

Suppose that the (countable torsion-free) group $G(j), j \geq 0$, and $K(j) \triangleleft G(j)$, have already been constructed so that $H \leq G(j), G(j)=H K(j), H \cap K(j)=M$ and, if $j \geq 1$, then $t_{j} g_{j} t_{j}^{-1}=z_{j}$ for some $t_{j} \in K(j)$ and $z_{j} \in Z$. The group $G(j+1)$, containing $G(j)$, is defined as the following HNN-extension:

$$
G(j+1)=\left\langle G(j), t_{j+1} \mid t_{j+1} g_{j+1} t_{j+1}^{-1}=z_{j+1}\right\rangle,
$$

where $z_{j+1} \in Z \subset H$ is the unique representative satisfying $g_{j+1} \in z_{j+1} K(j)$ in $G(j)$. Denote by $K(j+1) \triangleleft G(j+1)$ the normal closure of $\left\langle K(j), t_{j+1}\right\rangle$ in $G(j+1)$. Evidently the group $G(j+1)$ is countable and torsion-free, $H \leq G(j) \leq$ $G(j+1), G(j+1)=H K(j+1)$ and $H \cap K(j+1)=H \cap K(j)=M$ by Lemma 4.3.

Now, it is easy to verify that the group $G_{1}=\bigcup_{j=0}^{\infty} G(j)$ and its normal subgroup $K_{1}=\bigcup_{j=0}^{\infty} K(j)$ enjoy the required properties.

In the same way we can embed $G_{1}$ into a countable torsion-free group $G_{2}$, that has a normal subgroup $K_{2} \triangleleft G_{2}$, so that $G_{2}=H K_{2}, H \cap K_{2}=M$ and each element of $G_{1} \backslash\{1\}$ is conjugated in $G_{2}$ to a corresponding element of $Z$. Performing such a procedure infinitely many times we achieve the group $G=\bigcup_{i=1}^{\infty} G_{i}$ and a normal subgroup $K=\bigcup_{i=1}^{\infty} K_{i} \triangleleft G$ that satisfy the claims 1) and 2) of the lemma. It is easy to see that the claim 2) implies 3), thus the proof is finished. 


\section{Adding finite generation}

Theorem 5.1. Assume that $H$ is a countable torsion-free group and $M$ is a nontrivial normal subgroup of $H$. Let $F$ be an arbitrary non-elementary torsion-free word hyperbolic group. Then there exist a countable torsion-free group $Q$, containing $H$, and a normal subgroup $N \triangleleft Q$ with the following properties:

1. $H$ is malnormal in $Q$;

2. $Q=H \cdot N$ and $N \cap H=M$;

3. $N$ is a quotient of $F$;

4. the centralizer $C_{Q}(N)$ of $N$ in $Q$ is trivial;

5. for every $q \in Q$ there is $z \in H$ such that $q \stackrel{Q}{\sim} z$.

Proof. The group $Q$ will be constructed as a direct limit of relatively hyperbolic groups.

Step 0. Set $G(0)=H * F$ and $F(0)=F$; then $G(0)$ is hyperbolic relative to its subgroup $H$ and $F(0)$ is a suitable subgroup of $G(0)$ by Lemma 2.6. Let $N(0) \triangleleft G(0)$ be the normal closure of the subgroup $\langle M, F\rangle$ in $G(0)$. Evidently $G(0)=H \cdot N(0)$ and $H \cap N(0)=M$. Enumerate all the elements of $N(0)$ : $\left\{g_{0}, g_{1}, g_{2}, \ldots\right\}$, and of $G(0):\left\{q_{0}, q_{1}, q_{2}, \ldots\right\}$, in such a way that $g_{0}=q_{0}=1$.

Steps $0-i$. Assume the groups $G(j), j=0, \ldots, i, i \geq 0$, have been already constructed, so that

$1^{\circ}$. for each $1 \leq j \leq i$ there is an epimorphism $\psi_{j-1}: G(j-1) \rightarrow G(j)$ which is injective on (the image of) $H$ in $G(j-1)$. Denote $F(j)=\psi_{j-1}(F(j-1))$, $N(j)=\psi_{j-1}(N(j-1))$;

$2^{\circ}$. $G(j)$ is torsion-free and hyperbolic relative to (the image of) $H$, and $F(j) \leq$ $G(j)$ is a suitable subgroup, $j=0, \ldots, i$;

$3^{\circ} . G(j)=H \cdot N(j), N(j) \triangleleft G(j)$ and $H \cap N(j)=M, j=0, \ldots, i$;

$4^{\circ}$. the natural image $\bar{g}_{j}$ of $g_{j}$ in $G(j)$ belongs to $F(j), j=0, \ldots, i$;

$5^{\circ}$. there exists $z_{j} \in H$ such that $\bar{q}_{j} \stackrel{G(j)}{\sim} z_{j}, j=0, \ldots, i$, where $\bar{q}_{j}$ is the image of $q_{j}$ in $G(j)$.

Step $i+1$. Let $\hat{q}_{i+1} \in G(i), \hat{g}_{i+1} \in N(i)$ be the images of $q_{i+1}$ and $g_{i+1}$ in $G(i)$. First we construct the group $G(i+1 / 2)$, its normal subgroup $K_{i+1}$ and its element $t_{i+1}$ as follows.

If for some $f \in G(i), f \hat{q}_{i+1} f^{-1}=z \in H$, then set $G(i+1 / 2)=G(i)$, $K_{i+1}=N(i) \triangleleft G(i+1 / 2)$ and $t_{i+1}=1$.

Otherwise, $\hat{q}_{i+1}$ is a hyperbolic element of infinite order in $G(i)$. Since $G(i)$ is torsion-free, the elementary subgroup $E_{G(i)}\left(\hat{q}_{i+1}\right)$ is cyclic, thus $E_{G(i)}\left(\hat{q}_{i+1}\right)=$ 
$\langle h x\rangle$ for some $h \in H$ and $x \in N(i)$ (by $3^{\circ}$ ), and $\hat{q}_{i+1}=(h x)^{m}$ for some $m \in \mathbb{Z}$. Now, by Lemma 2.2, $G(i)$ is hyperbolic relative to $\{H,\langle h x\rangle\}$. Choose $y \in M$ so that $h y \neq 1$ and let $G(i+1 / 2)$ be the following HNN-extension of $G(i)$ :

$$
G(i+1 / 2)=\left\langle G(i), t_{i+1} \mid t_{i+1}(h x) t_{i+1}^{-1}=h y\right\rangle .
$$

The group $G(i+1 / 2)$ is torsion-free and hyperbolic relative to $H$ by Lemma 2.5 .

Let us now verify that the subgroup $F(i)$ is suitable in $G(i+1 / 2)$. Indeed, according to Lemma 2.7, there are two hyperbolic elements $f_{1}, f_{2} \in F(i)$ of infinite order in $G(i)$ such that $f_{l} \stackrel{G(i)}{\not} h x, f_{l} \stackrel{G(i)}{\not} h y, l=1,2$, and $f_{1} \stackrel{G(i)}{\not} f$. Then $f_{1} \stackrel{G(i+1 / 2)}{\not 2} f_{2}$ by Lemma 3.1. It remains to check that $f_{l}$ is a hyperbolic element of $G(i+1 / 2)$ for each $l=1,2$. Choose an arbitrary element $w \in H$ and observe $G(i)$

that $f_{l} \not z w$ (since $H$ is malnormal in $G(i)$ by Lemma 2.3, a non-trivial power of $f_{l}$ is conjugated to an element of $H$ if and only if $f_{l}$ is conjugated to an element of $H$ in $G(i)$, but the latter is impossible because $f_{l}$ is hyperbolic in $G(i)$ ). Applying Lemma 3.1 again, we get that $f_{l} \stackrel{G(i+1 / 2)}{\not} w$ for any $w \in H$. Hence $f_{1}, f_{2} \in F(i)$ are hyperbolic elements of infinite order in $G(i+1 / 2)$. The intersection $E_{G(i+1 / 2)}\left(f_{1}\right) \cap$ $E_{G(i+1 / 2)}\left(f_{2}\right)$ must be finite, since these groups are virtually cyclic (by Lemma 2.2 ), and $f_{1}$ is not commensurable with $f_{2}$ in $G(i+1 / 2)$. But $G(i+1 / 2)$ is torsion-free, therefore $E_{G(i+1 / 2)}\left(f_{1}\right) \cap E_{G(i+1 / 2)}\left(f_{2}\right)=\{1\}$. Thus $F(i)$ is a suitable subgroup of $G(i+1 / 2)$.

Lemma 4.3 assures that $H \cap K_{i+1}=M$ where $K_{i+1} \triangleleft G(i+1 / 2)$ is the normal closure of $\left\langle N(i), t_{i+1}\right\rangle$ in $G(i+1 / 2)$. Finally, note that

$$
t_{i+1} \hat{q}_{i+1} t_{i+1}^{-1}=t_{i+1}(h x)^{m} t_{i+1}^{-1}=(h y)^{m}=z \in H \text { in } G(i+1 / 2) .
$$

Now, that the group $G(i+1 / 2)$ has been constructed, set $T_{i+1}=\left\{\hat{g}_{i+1}, t_{i+1}\right\} \subset$ $K_{i+1}$ and define $G(i+1)$ as follows. Since $T_{i+1} \cdot F(i) \subset K_{i+1} \triangleleft G(i+1 / 2)$, we can apply Theorem 2.8 to find a group $G(i+1)$ and an epimorphism $\varphi_{i}: G(i+1 / 2) \rightarrow$ $G(i+1)$ such that $\varphi_{i}$ is injective on $H, G(i+1)$ is torsion-free and hyperbolic relative to (the image of) $H,\left\{\varphi_{i}\left(\hat{g}_{i+1}\right), \varphi_{i}\left(t_{i+1}\right)\right\} \subset \varphi_{i}(F(i)), \varphi_{i}(F(i))$ is a suitable subgroup of $G(i+1)$, and $\operatorname{ker}\left(\varphi_{i}\right) \leq K_{i+1}$. Denote by $\psi_{i}$ the restriction of $\varphi_{i}$ on $G(i)$. Then $\psi_{i}(G(i))=\varphi_{i}(G(i))=G(i+1)$ because $G(i+1 / 2)$ was generated by $G(i)$ and $t_{i+1}$, and according to the construction, $t_{i+1} \in \varphi_{i}(F(i)) \leq \varphi_{i}(G(i))$. Now, after defining $F(i+1)=\psi_{i}(F(i)), N(i+1)=\psi_{i}(N(i)), \bar{g}_{i+1}=\varphi_{i}\left(\hat{g}_{i+1}\right) \in F(i+1)$ and $z_{i+1}=\varphi_{i}(z) \in H$, we see that the conditions $1^{\circ}, 2^{\circ}, 4^{\circ}$ and $5^{\circ}$ hold in the case when $j=i+1$. The properties $G(i+1)=H \cdot N(i+1)$ and $N(i+1) \triangleleft G(i+1)$ 
are immediate consequences of their analogs for $G(i)$ and $N(i)$. Finally, observe that

$$
\begin{aligned}
\varphi_{i}^{-1}(H \cap N(i+1)) & =H \cdot \operatorname{ker}\left(\varphi_{i}\right) \cap N(i) \cdot \operatorname{ker}\left(\varphi_{i}\right) \\
& =\left(H \cap N(i) \cdot \operatorname{ker}\left(\varphi_{i}\right)\right) \cdot \operatorname{ker}\left(\varphi_{i}\right) \\
& \subseteq\left(H \cap K_{i+1}\right) \cdot \operatorname{ker}\left(\varphi_{i}\right)=M \cdot \operatorname{ker}\left(\varphi_{i}\right) .
\end{aligned}
$$

Therefore $H \cap N(i+1)=M$ and the condition $3^{\circ}$ holds for $G(i+1)$.

Let $Q=G(\infty)$ be the direct limit of the sequence $\left(G(i), \psi_{i}\right)$ as $i \rightarrow \infty$, and let $F(\infty)$ and $N=N(\infty)$ be the limits of the corresponding subgroups. Then $Q$ is torsion-free by $2^{\circ}, N \triangleleft Q, Q=H \cdot N$ and $H \cap N=M$ by $3^{\circ} . N \leq F(\infty)$ by $4^{\circ}$, and $5^{\circ}$ implies the condition 5 from the claim.

Since $F(0) \leq N(0)$ we get $F(\infty) \leq N$. Thus $N=F(\infty)$ is a homomorphic image of $F(0)=F$.

For any $i, j \in \mathbb{N} \cup\{\infty\}, i<j$, we have a natural epimorphism $\zeta_{i j}: G(i) \rightarrow$ $G(j)$ such that if $i<j<k$ then $\zeta_{j k} \circ \zeta_{i j}=\zeta_{i k}$. Take any $g \in G(0)$. Since $F=F(0)$ is finitely generated, using the properties of direct limits one can show that if $w=\zeta_{0 \infty}(g) \in C_{Q}(F(\infty))$ in $Q$, then $\zeta_{0 j}(g) \in C_{G(j)}(F(j))$ for some $j \in \mathbb{N}$. But $C_{G(j)}(F(j)) \leq E_{G(j)}(F(j))=\{1\}$ (by formulas (2.2) and (2.3)) because $F(j)$ is a suitable subgroup of $G(j)$, hence $w=\zeta_{j \infty}\left(\zeta_{0 j}(g)\right)=1$, that is, $C_{Q}(F(\infty))=C_{Q}(N)=\{1\}$. This concludes the proof.

The next statement is well known.

Lemma 5.2. Assume $G$ is a group and $N \triangleleft G$ is a normal subgroup such that $C_{G}(N) \subseteq N$, where $C_{G}(N)$ is the centralizer of $N$ in $G$. Then the quotient-group $G / N$ embeds into the outer automorphism group $\operatorname{Out}(N)$.

Proof. The action of $G$ on $N$ by conjugation induces a natural homomorphism $\varphi$ from $G$ to the automorphism group $\operatorname{Aut}(N)$ of $N$. Since $\varphi(N)$ is exactly the group of inner automorphisms $\operatorname{Inn}(N)$ of $N$, one can define a new homomorphism $\bar{\varphi}: G / N \rightarrow$ $\operatorname{Out}(N)=\operatorname{Aut}(N) / \operatorname{Inn}(N)$ in the natural way: $\bar{\varphi}(g N)=\varphi(g) \operatorname{Inn}(N)$ for every $g N \in G / N$. It remains to check that $\bar{\varphi}$ is injective, i.e., if $g \in G \backslash N$ then $\bar{\varphi}(g N) \neq 1$ in $\operatorname{Out}(N)$; or, equivalently, $\varphi(g) \notin \operatorname{Inn}(N)$. Indeed, otherwise there would exist $a \in N$ such that $g h g^{-1}=a h a^{-1}$ for every $h \in N$, thus $N \not \ngtr a^{-1} g \in C_{G}(N)$, contradicting the assumptions.

Note that for an arbitrary group $N$, any subgroup $C \leq \operatorname{Out}(N)$ naturally acts on the set of conjugacy classes $\mathfrak{C}(N)$ of the group $N$.

Theorem 5.3. For any $n \in \mathbb{N}, n \geq 2$, and an arbitrary countable group $C, C$ can be isomorphically embedded into the outer automorphism group $\operatorname{Out}(N)$ of a group $N$ satisfying the following conditions: 
- $N$ is torsion-free;

- $N$ is generated by two elements;

- $N$ has $(n \mathrm{CC})$ and the natural action of $C$ on $\mathfrak{C}(N)$ is trivial;

- $N$ is 2-boundedly simple.

Proof. By Lemma 4.2 we can find a countable torsion-free group $H$ and its normal subgroup $M$ enjoying the properties (i)-(iv) from its claim. Now, if $F$ denotes the free group of rank 2, we can obtain a countable torsion-free group $Q$ together with its normal subgroup $N$ that satisfy the conditions $1-5$ from the statement of Theorem 5.1.

Then $N$ is torsion-free and generated by two elements (as a quotient of $F$ ). Condition 2 implies that $Q / N \cong H / M \cong C$ and, by 4 and Lemma 5.2, $C$ embeds into the group $\operatorname{Out}(N)$.

Using property 5 , for each $g \in N$ we can find $u \in Q$ and $z \in H$ such that $u g u^{-1}=z \in N \cap H=M$. Since $Q=H N$, there are $h \in H$ and $x \in N$ such that $u=h x$. Since $z, h^{-1} z h \in M$ and the action of $H$ on $M$ preserves the conjugacy classes of $M$, there is $r \in M$ such that $r h^{-1} z h r^{-1}=z$, hence $z=r h^{-1} u g u^{-1}\left(r h^{-1}\right)^{-1}=r x g x^{-1} r^{-1}$, where $v=r x \in N$. Thus for every $g \in N$ there is $v \in N$ such that $v g v^{-1} \in M$. Evidently, this implies that $N$ is also 2-boundedly simple. Since $M$ has (nCC), the number of conjugacy classes in $N$ will be at most $n$.

Suppose $x_{1}, x_{2} \in M$ and $x_{1} \stackrel{M}{\nsim} x_{2}$. Then $x_{1} \stackrel{H}{\nsim} x_{2}$ (by the property (iii) from the claim of Lemma 4.2), and since $H$ is malnormal in $Q$ we get $x_{1} \underset{\sim}{Q} x_{2}$. Hence $x_{1} \stackrel{N}{\sim} x_{2}$, i.e., $N$ also enjoys ( $n \mathrm{CC}$ ).

The fact that the natural action of $C$ on $\mathfrak{C}(N)$ is trivial follows from the same property for the action of $H$ on $\mathfrak{C}(M)$ and the malnormality of $H$ in $Q$.

Now, let us proceed with the

Proof of Theorem 1.4. First we apply Lemma 4.4 to construct a group $G$ and a normal subgroup $K \triangleleft G$ according to its claim. Now, by Theorem 5.1, there is a group $Q$, having a normal subgroup $N \triangleleft Q$ such that $G$ is malnormal in $Q, Q=G N$, $G \cap N=K, \operatorname{rank}(N) \leq 2$ (if one takes the free group of rank 2 as $F$ ) and every element $q \in Q$ is conjugated (in $Q$ ) to an element of $G$. By claim 2) of Lemma 4.4, $K$ has (2CC), and an argument, similar to the one used in the proof of Theorem 5.3, shows that $N$ will also have $(2 \mathrm{CC})$. Consequently, $\operatorname{rank}(N)>1$ because $N$ is torsion-free, hence $\operatorname{rank}(N)=2$.

Since $G=H K$ and $H \cap K=M$ we have $Q=H K N=H N$ and $H \cap N=$ $H \cap K=M$. Since $Q / N \cong H / M$ and $N$ can be generated by two elements, we can conclude that $\operatorname{rank}(Q) \leq \operatorname{rank}(H / M)+2$. 
Consider arbitrary $x, y \in Q \backslash\{1\}$ and suppose that $\varphi(x) \stackrel{Q / N}{\sim} \varphi(y)$. By Theorem 5.1, there are $w, z \in G \backslash\{1\}$ such that $x \stackrel{Q}{\sim} w$ and $y \stackrel{Q}{\sim} z$. Therefore $\varphi(w) \stackrel{Q / N}{\sim} \varphi(z)$, hence the images of $w$ and $z$ in $G / K$ are also conjugate. By claim 3) of Lemma 4.4, $\underset{\stackrel{G}{\sim}}{\sim}$, implying $x \stackrel{Q}{\sim} y$.

Theorem 1.4 provides an alternative way of obtaining torsion-free groups that have finitely many conjugacy classes: for any countable group $C$ we can choose a free group $H$ of countable rank and a normal subgroup $\{1\} \neq M \triangleleft H$ so that $H / M \cong C$, and then apply Theorem 1.4 to the pair $(H, M)$ to get

Corollary 5.4. Assume that $n \in \mathbb{N}, n \geq 2$, and $C$ is a countable group that contains exactly $(n-1)$ distinct conjugacy classes. Then there exists a torsion-free group $Q$ and $N \triangleleft Q$ such that

- $Q / N \cong C$;

- $N$ has (2CC) and $Q$ has ( $n \mathrm{CC})$;

- $\operatorname{rank}(N)=2$ and $\operatorname{rank}(Q) \leq \operatorname{rank}(C)+2$.

Corollary 5.5. The group $G_{1}$, given by presentation (1.1), can be isomorphically embedded into a 2-generated torsion-free group $Q$ satisfying (4CC) in such a way that $t \stackrel{Q}{\sim} t^{-1}$.

Proof. Denote by $K$ the kernel of the homomorphism $\varphi: G_{1} \rightarrow \mathbb{Z}_{3}$, for which $\varphi(a)=0$ and $\varphi(t)=1$, where $\mathbb{Z}_{3}$ is the group of integers modulo 3. Now, apply Theorem 1.4 to the pair $\left(G_{1}, K\right)$ to find the group $Q$, containing $G_{1}$, and the normal subgroup $N \triangleleft Q$ from its claim. Since $Q / N \cong G_{1} / K \cong \mathbb{Z}_{3}$ has (3CC), the group $Q$ will have (4CC). We also have $t \stackrel{Q}{x} t^{-1}$ because the images of $t$ and $t^{-1}$ are not conjugate in $Q / N$.

Choose an element $q_{1} \in Q \backslash N$. Then $q_{2}=q_{1}^{3} \in N \backslash\{1\}$ and since $N$ is 2-generated and has (2CC), there is $q_{3} \in N$ such that $N=\left\langle q_{2}, q_{3}\right\rangle$ in $Q$. As $Q / N$ is generated by the image of $q_{1}$, the group $Q$ will be generated by $\left\{q_{1}, q_{2}, q_{3}\right\}$, and, consequently, by $\left\{q_{1}, q_{3}\right\}$.

\section{Combinatorics of paths in relatively hyperbolic groups}

Let $G$ be a group hyperbolic relative to a family of proper subgroups $\left\{H_{\lambda}\right\}_{\lambda \in \Lambda}$, and let $\mathcal{X}$ be a finite symmetrized relative generating set of $G$. Denote $\mathscr{H}=$ $\bigcup_{\lambda \in \Lambda}\left(H_{\lambda} \backslash\{1\}\right)$. 
For a combinatorial path $p$ in the Cayley graph $\Gamma(G, \mathcal{X} \cup \mathscr{H}$ ) (of $G$ with respect to $\mathcal{X} \cup \mathscr{H}) p_{-}, p_{+}, \mathrm{L}(p)$, and $\operatorname{Lab}(p)$ will denote the initial point, the ending point, the length (that is, the number of edges) and the label of $p$ respectively. $p^{-1}$ will be the path obtained from $p$ by following it in the reverse direction. Further, if $\Omega$ is a subset of $G$ and $g \in\langle\Omega\rangle \leq G$, then $|g|_{\Omega}$ will be used to denote the length of a shortest word in $\Omega^{ \pm 1}$ representing $g$.

We will be using the following terminology from [20]. Suppose $q$ is a path in $\Gamma(G, \mathcal{X} \cup \mathscr{H})$. A subpath $p$ of $q$ is called an $H_{\lambda}$-component for some $\lambda \in \Lambda$ (or simply a component) of $q$, if the label of $p$ is a word in the alphabet $H_{\lambda} \backslash\{1\}$ and $p$ is not contained in a bigger subpath of $q$ with this property.

Two components $p_{1}, p_{2}$ of a path $q$ in $\Gamma(G, \mathcal{X} \cup \mathscr{H})$ are called connected if they are $H_{\lambda}$-components for the same $\lambda \in \Lambda$ and there exists a path $c$ in $\Gamma(G, \mathcal{X} \cup \mathscr{H})$ connecting a vertex of $p_{1}$ to a vertex of $p_{2}$ such that $\operatorname{Lab}(c)$ entirely consists of letters from $H_{\lambda}$. In algebraic terms this means that all vertices of $p_{1}$ and $p_{2}$ belong to the same coset $g H_{\lambda}$ for a certain $g \in G$. We can always assume $c$ to have length at most 1 , as every nontrivial element of $H_{\lambda}$ is included in the set of generators. An $H_{\lambda}$-component $p$ of a path $q$ is called isolated if no other $H_{\lambda}$-component of $q$ is connected to $p$.

The next statement is a particular case of Lemma 2.27 from [20]; we shall formulate it in a slightly more general form, as it appears in [18, Lemma 2.7]:

Lemma 6.1. Suppose that a group $G$ is hyperbolic relative to a family of subgroups $\left\{H_{\lambda}\right\}_{\lambda \in \Lambda}$. Then there exists a finite subset $\Omega \subseteq G$ and a constant $K \in \mathbb{N}$ such that the following holds. Let $q$ be a cycle in $\Gamma(G, \mathcal{X} \cup \mathscr{H}), p_{1}, \ldots, p_{k}$ be a collection of isolated components of $q$ and $g_{1}, \ldots, g_{k}$ be the elements of $G$ represented by $\operatorname{Lab}\left(p_{1}\right), \ldots, \operatorname{Lab}\left(p_{k}\right)$ respectively. Then $g_{1}, \ldots, g_{k}$ belong to the subgroup $\langle\Omega\rangle \leq G$ and the word lengths of $g_{i}$ 's with respect to $\Omega$ satisfy

$$
\sum_{i=1}^{k}\left|g_{i}\right|_{\Omega} \leq K \mathrm{~L}(q) .
$$

Definition. Suppose that $m \in \mathbb{N}$ and $\Omega$ is a finite subset of $G$. Define $\mathcal{W}(\Omega, m)$ to be the set of all words $W$ over the alphabet $\mathcal{X} \cup \mathscr{H}$ that have the following form:

$$
W \equiv x_{0} h_{0} x_{1} h_{1} \ldots x_{l} h_{l} x_{l+1}
$$

where $l \in \mathbb{Z}, l \geq-2$ (if $l=-2$ then $W$ is the empty word; if $l=-1$ then $W \equiv x_{0}$ ), $h_{i}$ and $x_{i}$ are considered as single letters and

1) $x_{i} \in \mathcal{X} \cup\{1\}, i=0, \ldots, l+1$, and for each $i=0, \ldots, l$, there exists $\lambda(i) \in \Lambda$ such that $h_{i} \in H_{\lambda(i)}$;

2) if $\lambda(i)=\lambda(i+1)$ then $x_{i+1} \notin H_{\lambda(i)}$ for each $i=0, \ldots, l-1$; 
3) $h_{i} \notin\left\{\left.h \in\langle\Omega\rangle|| h\right|_{\Omega} \leq m\right\}, i=0, \ldots, l$.

Choose the finite subset $\Omega \subset G$ and the constant $K>0$ according to the claim of Lemma 6.1.

Recall that a path $q$ in $\Gamma(G, \mathcal{X} \cup \mathscr{H})$ is said to be without backtracking if all of its components are isolated.

Lemma 6.2. Let $q$ be a path in the Cayley graph $\Gamma(G, \mathcal{X} \cup \mathcal{H})$ with $\operatorname{Lab}(q) \in$ $\mathcal{W}(\Omega, m)$ and $m \geq 5 K$. Then $q$ is without backtracking.

Proof. Assume the contrary to the claim. Then one can choose a path $q$ providing a counterexample of the smallest possible length. Thus if $p_{1}, \ldots, p_{l}$ is the (consecutive) list of all components of $q$ then $l \geq 2, p_{1}$ and $p_{l}$ must be connected $H_{\lambda^{\prime}}$ components, for some $\lambda^{\prime} \in \Lambda$, the components $p_{2}, \ldots, p_{l-1}$ must be isolated, and $q$ starts with $p_{1}$ and ends with $p_{l}$. Since $\operatorname{Lab}(q) \in \mathcal{W}(\Omega, m)$ we have $\operatorname{L}(q) \leq 2 l-1$.

If $l=2$ then the $(\mathcal{X} \cup\{1\})$-letter between $p_{1}$ and $p_{2}$ would belong to $H_{\lambda^{\prime}}$ contradicting the property 2$)$ from the definition of $\mathcal{W}(\Omega, m)$.

Therefore $l \geq 3$. Since $p_{1}$ and $p_{l}$ are connected, there exists a path $v$ in $\Gamma(G, \mathcal{X} \cup$ $\mathscr{H}$ ) between $\left(p_{l}\right)_{-}$and $\left(p_{1}\right)_{+}$with $\operatorname{Lab}(v) \in H_{\lambda^{\prime}}$ (thus we can assume that $\left.\mathrm{L}(v) \leq 1\right)$. Denote by $\hat{q}$ the subpath of $q$ starting with $\left(p_{1}\right)_{+}$and ending with $\left(p_{l}\right)_{-}$. Note that $\mathrm{L}(\hat{q})=\mathrm{L}(q)-2 \leq 2 l-3$, and $p_{2}, \ldots, p_{l-1}$ is the list of components of $\hat{q}$, all of which are isolated. If one of them were connected to $v$ it would imply that it is connected to $p_{1}$ contradicting with the minimality of $q$. Hence the cycle $o=\hat{q} v$ possesses $k=l-2 \geq 1$ isolated components, which represent elements $h_{1}, \ldots, h_{k} \in \mathcal{H}$. Consequently, applying Lemma 6.1 one obtains that $h_{i} \in\langle\Omega\rangle, i=1, \ldots, k$, and

$$
\sum_{i=1}^{k}\left|h_{i}\right|_{\Omega} \leq K \mathrm{~L}(o) \leq K(\mathrm{~L}(\hat{q})+1) \leq K(2 l-2) .
$$

By the condition 3) from the definition of $\mathcal{W}(\Omega, m)$ one has $\left|h_{i}\right|_{\Omega}>m \geq 5 K$ for each $i=1, \ldots, k$. Hence

$$
k \cdot 5 K \leq \sum_{i=1}^{k}\left|h_{i}\right|_{\Omega} \leq K(2 l-2), \quad \text { or } \quad 5 \leq \frac{2 l-2}{k},
$$

which contradicts the inequality $k \geq l-2$.

Definition. Consider an arbitrary cycle $o=r q r^{\prime} q^{\prime}$ in $\Gamma(G, \mathcal{X} \cup \mathcal{H})$, where $\operatorname{Lab}(q)$ and $\operatorname{Lab}\left(q^{\prime}\right)$ belong to $\mathcal{W}(\Omega, m)$. Let $p$ be a component of $q$ (or $\left.q^{\prime}\right)$. We will say that $p$ is regular if it is not an isolated component of $o$. If $m \geq 5 K$, and hence $q$ and $q^{\prime}$ are without backtracking by Lemma 6.2, this means that $p$ is either connected to some component of $q^{\prime}$ (respectively $q$ ), or to a component of $r$ or $r^{\prime}$. 
Lemma 6.3. In the above notations, suppose that $m \geq 7 K$ and denote $C=$ $\max \left\{\mathrm{L}(r), \mathrm{L}\left(r^{\prime}\right)\right\}$. Then

(a) if $C \leq 1$ then every component of $q$ or $q^{\prime}$ is regular;

(b) if $C \geq 2$ then each of $q$ and $q^{\prime}$ can have at most $4 C$ components which are not regular.

(c) if $l$ is the number of components of $q$, then at least $(l-6 C)$ of components of $q$ are connected to components of $q^{\prime}$; and two distinct components of $q$ can not be connected to the same component of $q^{\prime}$. Similarly for $q^{\prime}$.

Proof. Assume the contrary to (a). Then one can choose a cycle $o=r q r^{\prime} q^{\prime}$ with $\mathrm{L}(r), \mathrm{L}\left(r^{\prime}\right) \leq 1$, having at least one isolated component on $q$ or $q^{\prime}$, and such that $\mathrm{L}(q)+\mathrm{L}\left(q^{\prime}\right)$ is minimal. Clearly the latter condition implies that each component of $q$ or $q^{\prime}$ is an isolated component of $o$. Therefore $q$ and $q^{\prime}$ together contain $k$ distinct isolated components of $o$, representing elements $h_{1}, \ldots, h_{k} \in \mathcal{H}$, where $k \geq 1$ and $k \geq(\mathrm{L}(q)-1) / 2+\left(\mathrm{L}\left(q^{\prime}\right)-1\right) / 2$. Applying Lemma 6.1 we obtain $h_{i} \in\langle\Omega\rangle$, $i=1, \ldots, k$, and

$$
\sum_{i=1}^{k}\left|h_{i}\right|_{\Omega} \leq K \mathrm{~L}(o) \leq K\left(\mathrm{~L}(q)+\mathrm{L}\left(q^{\prime}\right)+2\right) .
$$

Recall that $\left|h_{i}\right|_{\Omega}>m \geq 7 K$ by the property 3) from the definition of $\mathcal{W}(\Omega, m)$. Therefore $\sum_{i=1}^{k}\left|h_{i}\right|_{\Omega} \geq k \cdot 7 K$, implying

$$
7 \leq \frac{2}{k}\left(\frac{\mathrm{L}(q)}{2}+\frac{\mathrm{L}\left(q^{\prime}\right)}{2}+1\right) \leq \frac{2}{k}\left(\frac{\mathrm{L}(q)-1}{2}+\frac{\mathrm{L}\left(q^{\prime}\right)-1}{2}+2\right) \leq 6,
$$

which yields a contradiction.

Let us prove (b). Suppose that $C \geq 2$ and $q$ contains more than $4 C$ isolated components of $o$. We shall consider two cases:

Case 1 . No component of $q$ is connected to a component of $q^{\prime}$. Then a component of $q$ or $q^{\prime}$ can be regular only if it is connected to a component of $r$ or $r^{\prime}$. Since, by Lemma 6.2, $q$ and $q^{\prime}$ are without backtracking, two distinct components of $q$ or $q^{\prime}$ can not be connected to the same component of $r$ (or $r^{\prime}$ ). Hence $q$ and $q^{\prime}$ together can contain at most $2 C$ regular components. Thus the cycle $o$ has $k$ isolated components, representing elements $h_{1}, \ldots, h_{k} \in \mathscr{H}$, where $k \geq 4 C>4$ and $k \geq$ $(\mathrm{L}(q)-1) / 2+\left(\mathrm{L}\left(q^{\prime}\right)-1\right) / 2-2 C$. By Lemma 6.1, $h_{i} \in\langle\Omega\rangle$ for each $i=1, \ldots, k$, and $\sum_{i=1}^{k}\left|h_{i}\right|_{\Omega} \leq K\left(\mathrm{~L}(q)+\mathrm{L}\left(q^{\prime}\right)+2 C\right)$. Once again we can use the property 3$)$ 
from the definition of $\mathcal{W}(\Omega, m)$ to achieve

$$
\begin{aligned}
7 & \leq \frac{2}{k}\left(\frac{\mathrm{L}(q)}{2}+\frac{\mathrm{L}\left(q^{\prime}\right)}{2}+\frac{2 C}{2}\right) \leq \frac{2}{k}\left(\frac{\mathrm{L}(q)-1}{2}+\frac{\mathrm{L}\left(q^{\prime}\right)-1}{2}-2 C+1+3 C\right) \\
& \leq \frac{2}{k}\left(\frac{\mathrm{L}(q)-1}{2}+\frac{\mathrm{L}\left(q^{\prime}\right)-1}{2}-2 C\right)+\frac{2}{k}+\frac{6 C}{k} \leq 2+\frac{1}{2}+\frac{3}{2}=4,
\end{aligned}
$$

yielding a contradiction.

Case 2. The path $q$ has at least one component which is connected to a component of $q^{\prime}$. Let $p_{1}, \ldots, p_{l}$ denote the sequence of all components of $q$. By part (a), if $p_{s}$ and $p_{t}, 1 \leq s \leq t \leq l$, are connected to components of $q^{\prime}$, then for any $j, s \leq j \leq t$, $p_{j}$ is connected to some component of $q^{\prime}$ (because $q$ is without backtracking by Lemma 6.2). We can take $s$ (respectively $t$ ) to be minimal (respectively maximal) possible. Consequently $p_{1}, \ldots, p_{s-1}, p_{t+1}, \ldots, p_{l}$ will contain the set of all isolated components of $o$ that belong to $q$, and none of these components will be connected to a component of $q^{\prime}$.

Without loss of generality we may assume that $s-1 \geq 4 C / 2=2 C$. Since $p_{s}$ is connected to some component $p^{\prime}$ of $q^{\prime}$, there exists a path $v$ in $\Gamma(G, \mathcal{X} \cup \mathcal{H})$ satisfying $v_{-}=\left(p_{s}\right)_{-}, v_{+}=p_{+}^{\prime}, \operatorname{Lab}(v) \in \mathscr{H} \cup\{1\}, \mathrm{L}(v) \leq 1$. Let $\bar{q}$ (respectively $\bar{q}^{\prime}$ ) denote the subpath of $q$ (respectively $q^{\prime}$ ) from $q_{-}$to $\left(p_{s}\right)_{-}$(respectively from $p_{+}^{\prime}$ to $q_{+}^{\prime}$ ). Consider a new cycle $\bar{o}=r \bar{q} v \bar{q}^{\prime}$. Reasoning as before, one can show that $\bar{o}$ possesses $k$ isolated components, where $k \geq 2 C \geq 4$ and $k \geq(\mathrm{L}(\bar{q})-1) / 2+$ $\left(\mathrm{L}\left(\bar{q}^{\prime}\right)-1\right) / 2-C-1$. Now, an application of Lemma 6.1 to the cycle $\bar{o}$ together with the property 3$)$ from the definition of $\mathcal{W}(\Omega, m)$ will lead to a contradiction as before.

By the symmetry, the statement (b) of the lemma also holds for $q^{\prime}$.

The claim (c) follows from (b) and the estimate $\mathrm{L}(r)+\mathrm{L}\left(r^{\prime}\right) \leq 2 C$ because if two different components $p$ and $\bar{p}$ of $q$ were connected to the same component of some path in $\Gamma(G, \mathcal{X} \cup \mathscr{H})$, then $p$ and $\bar{p}$ would also be connected with each other, which would contradict Lemma 6.2 .

Lemma 6.4. In the previous notations, let $m \geq 7 K, C=\max \left\{\mathrm{L}(r), \mathrm{L}\left(r^{\prime}\right)\right\}$, and let $p_{1}, \ldots, p_{l}, p_{1}^{\prime}, \ldots, p_{l}^{\prime}$, be the consecutive lists of the components of $q$ and $q^{-1}$ respectively. If $l \geq 12 \max \{C, 1\}+2$, then there are indices $s, t, s^{\prime} \in \mathbb{N}$ such that $1 \leq s \leq 6 C+1, l-6 \max \{C, 1\} \leq t \leq l$ and for every $i \in\{0,1, \ldots, t-s\}$, the component $p_{s+i}$ of $q$ is connected to the component $p_{s^{\prime}+i}^{\prime}$ of $q^{\prime}$.

Proof. By part (c) of Lemma 6.3, there exists $s \leq 6 C+1$ such that the component $p_{s}$ is connected to a component $p_{s^{\prime}}^{\prime}$ for some $s^{\prime} \in\left\{1, \ldots, l^{\prime}\right\}$. Thus there is a path $r_{1}$ between $\left(p_{s^{\prime}}^{\prime}\right)_{+}$and $\left(p_{s}\right)_{+}$with $\mathrm{L}\left(r_{1}\right) \leq 1$. Consider a new cycle $o_{1}=r_{1} q_{1} r^{\prime} q_{1}^{\prime}$ where $q_{1}$ is the segment of $q$ from $\left(p_{s}\right)_{+}$to $q_{+}=r_{-}^{\prime}$ and $q_{1}^{\prime}$ is the segment of $q^{\prime}$ from $q_{-}^{\prime}=r_{+}^{\prime}$ to $\left(p_{s^{\prime}}^{\prime}\right)_{+}$. 
Observe that $p_{s+1}, \ldots, p_{l}$ is the list of all components of $q_{1}$ and $l-s \geq l-$ $6 C-1 \geq 6 \max \{1, C\}+1$, hence, according to part (c) of Lemma 6.3 applied to $o_{1}$, there is $t \geq l-6 \max \{1, C\}>s$ such that $p_{t}$ is connected to $p_{t^{\prime}}^{\prime}$ by means of a path $r_{1}^{\prime}$, where $s^{\prime}+1 \leq t^{\prime} \leq l^{\prime},\left(r_{1}^{\prime}\right)_{-}=\left(p_{t}\right)_{+},\left(r_{1}^{\prime}\right)_{+}=\left(p_{t^{\prime}}^{\prime}\right)_{+}$and $\mathrm{L}\left(r_{1}^{\prime}\right) \leq 1$. Consider the cycle $o_{2}=r_{1} q_{2} r_{1}^{\prime} q_{2}^{\prime}$ in which $q_{2}$ and $q_{2}^{\prime}$ are the segments of $q_{1}$ and $q_{1}^{\prime}$ from $\left(p_{s}\right)_{+}=\left(r_{1}\right)_{+}$to $\left(p_{t}\right)_{+}$and from $\left(p_{t^{\prime}}^{\prime}\right)_{+}$to $\left(p_{s^{\prime}}^{\prime}\right)_{+}=\left(r_{1}\right)_{-}$respectively (see Figure 1).

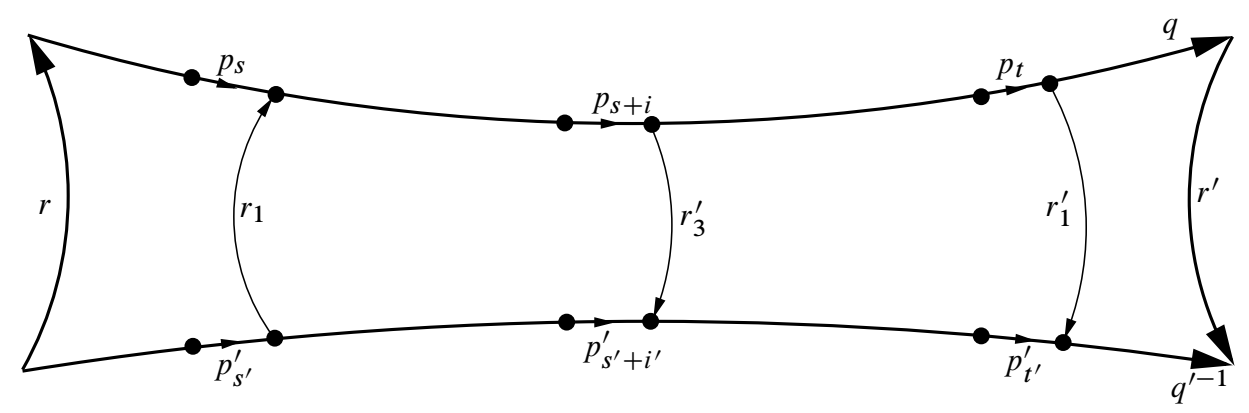

Figure 1

Note that $p_{s+1}, \ldots, p_{t}$ is the list of all components of $q_{2}$ and $p_{s^{\prime}+1}^{\prime}, \ldots, p_{t^{\prime}}^{\prime}$ is the list of all components of $q_{2}^{\prime-1}$. The cycle $o_{2}$ satisfies the assumptions of part (a) of Lemma 6.3, therefore for every $i \in\{1, \ldots, t-s\}$ there exists $i^{\prime} \in\left\{1, \ldots, t^{\prime}-s^{\prime}\right\}$ such that $p_{s+i}$ is connected to $p_{s^{\prime}+i^{\prime}}^{\prime}\left(p_{s+i}\right.$ can not be connected to $r_{1}\left[r_{1}^{\prime}\right]$ because in this case it would be connected to $p_{s}\left[p_{t}\right]$, but $q$ is without backtracking by Lemma 6.2).

It remains to show that $i^{\prime}=i$ for every such $i$. Indeed, if $i^{\prime}<i$ for some $i \in\{1, \ldots, t-s\}$ then one can consider the cycle $o_{3}=r_{1} q_{3} r_{3}^{\prime} q_{3}^{\prime}$, where $q_{3}$ and $q_{3}^{\prime}$ are segments of $q_{2}$ and $q_{2}^{\prime}$ from $\left(q_{2}\right)_{-}=\left(r_{1}\right)_{+}$to $\left(p_{s+i}\right)_{+}$and from $\left(p_{s^{\prime}+i^{\prime}}^{\prime}\right)_{+}$to $\left(q_{2}^{\prime}\right)_{+}=$ $\left(r_{1}\right)_{-}$respectively, and $\left(r_{3}^{\prime}\right)_{-}=\left(q_{3}\right)_{+},\left(r_{3}^{\prime}\right)_{+}=\left(q_{3}^{\prime}\right)_{-}, \mathrm{L}\left(r_{3}^{\prime}\right) \leq 1$. According to part (a) of Lemma 6.3, each of the components $p_{s+1}, \ldots, p_{s+i}$ of $q_{3}$ must be connected to one of $p_{s^{\prime}+1}^{\prime}, \ldots, p_{s^{\prime}+i^{\prime}}^{\prime}$. Hence, since $i^{\prime}<i$, two distinct components of $q_{3}$ will be connected to the same component of $q_{3}^{\prime-1}$, which is impossible by part (c) of Lemma 6.3.

The inequality $i^{\prime}>i$ would lead to a contradiction after an application of a symmetric argument to $q_{3}^{\prime}$. Therefore $i^{\prime}=i$ and the lemma is proved.

Lemma 6.5. In the above notations, let $m \geq 7 K$ and $C=\max \left\{\mathrm{L}(r), \mathrm{L}\left(r^{\prime}\right)\right\}$. For any positive integer $d$ there exists a constant $L=L(C, d) \in \mathbb{N}$ such that if $\mathrm{L}(q) \geq L$ then there are $d$ consecutive components $p_{s}, \ldots, p_{s+d-1}$ of $q$ and $p_{s^{\prime}}^{\prime}, \ldots, p_{s^{\prime}+d-1}^{\prime}$ of $q^{\prime-1}$, so that $p_{s+i}$ is connected to $p_{s^{\prime}+i}^{\prime}$ for each $i=0, \ldots, d-1$. 
Proof. Choose the constant $L$ so that $(L-1) / 2 \geq 12 \max \{C, 1\}+2+d$. Let $p_{1}, \ldots, p_{l}$ be the consecutive list all components of $q$. Since $\operatorname{Lab}(q) \in \mathcal{W}(\Omega, m)$, we have $l \geq(L-1) / 2$ (due to the form of any word from $\mathcal{W}(\Omega, m)$ ). Thus we can apply Lemma 6.4 to find indices $s, t$ from its claim. By the choice of $s$ and $t$, and the estimate on $l$, we have $t-s \geq d+1$, yielding the statement of the lemma.

Corollary 6.6. Let $G$ be a group hyperbolic relative to a family of proper subgroups $\left\{H_{\lambda}\right\}_{\lambda \in \Lambda}$. Suppose that $a \in H_{\lambda_{0}}$, for some $\lambda_{0} \in \Lambda$, is an element of infinite order, and $x_{1}, x_{2} \in G \backslash H_{\lambda_{0}}$. Then there exists $k \in \mathbb{N}$ such that $g=a^{k_{1}} x_{1} a^{k_{2}} x_{2}$ is a hyperbolic element of infinite order in $G$ whenever $\left|k_{1}\right|,\left|k_{2}\right| \geq k$.

Proof. Without loss of generality we can assume that $x_{1}, x_{2} \in \mathcal{X}$, since relative hyperbolicity does not depend on the choice of the finite relative generating set ([20, Theorem 2.34]). Choose the finite subset $\Omega \subset G$ and the constant $K \in \mathbb{N}$ according to the claim of Lemma 6.1, and set $m=7 \mathrm{~K}$. As the order of $a$ is infinite, there is $k \in \mathbb{N}$ such that $a^{k^{\prime}} \notin\left\{\left.h \in\langle\Omega\rangle|| h\right|_{\Omega} \leq m\right\}$ whenever $\left|k^{\prime}\right| \geq k$. Assume that $\left|k_{1}\right|,\left|k_{2}\right| \geq k$.

Suppose, first, that $g^{l}=1$ for some $l \in \mathbb{N}$. Consider the cycle $o=r q r^{\prime} q^{\prime}$ in $\Gamma(G, \mathcal{X} \cup \mathscr{H})$ where $q_{-}=q_{+}=1, \operatorname{Lab}(q) \equiv\left(a^{k_{1}} x_{1} a^{k_{2}} x_{2}\right)^{l} \in \mathcal{W}(\Omega, m)\left(a^{k_{j}}\right.$ are considered as single letters from the alphabet $\mathcal{X} \cup \mathscr{H})$ and $r, r^{\prime}, q^{\prime}$ are trivial paths (consisting of a single point). Then, by part (a) of Lemma 6.3, every component of $q$ must be regular in $o$, which is impossible since $q$ is without backtracking according to Lemma 6.2. Hence $g$ has infinite order in $G$.

Suppose, now, that there exists $\lambda^{\prime} \in \Lambda, u \in H_{\lambda^{\prime}}$ and $y \in G$ such that $y g y^{-1}=u$. Denote $C=|y| x \cup \mathscr{H}$. Since element $u \in G$ has infinite order, there exists $l \in \mathbb{N}$ such that $2 l \geq 6 C+2$ and $u^{l} \notin\left\{\left.h \in\langle\Omega\rangle|| h\right|_{\Omega} \leq m\right\}$. The equality $y g^{l} y^{-1} u^{-l}=1$ gives rise to the cycle $o=r q r^{\prime} q^{\prime}$ in $\Gamma(G, X \cup \mathscr{H})$, where $r$ and $r^{\prime}$ are paths of length $C$ whose labels represent $y$ in $G, r_{-}=1, q_{-}=r_{+}=y, \operatorname{Lab}(q) \equiv\left(a^{k_{1}} x_{1} a^{k_{2}} x_{2}\right)^{l} \in$ $\mathcal{W}(\Omega, m), r_{-}^{\prime}=q_{+}, q_{-}^{\prime}=r_{+}^{\prime}=y\left(a^{k_{1}} x_{1} a^{k_{2}} x_{2}\right)^{l} y^{-1}$ and $\operatorname{Lab}\left(q^{\prime}\right) \equiv u^{-l} \in$ $\mathcal{W}(\Omega, m), \mathrm{L}\left(q^{\prime}\right)=1$. By part (c) of Lemma 6.3, at least $2 l-6 C \geq 2$ distinct components of $q$ must be connected to distinct components of $q^{\prime}$, which is impossible as $q^{\prime}$ has only one component. The contradiction shows that $g$ must be a hyperbolic element of $G$.

Lemma 6.7. Let $G$ be a torsion-free group hyperbolic relative to a family of proper subgroups $\left\{H_{\lambda}\right\}_{\lambda \in \Lambda}, a \in H_{\lambda_{0}} \backslash\{1\}$, for some $\lambda_{0} \in \Lambda$, and $t, u \in G \backslash H_{\lambda_{0}}$. Suppose that there exists $\hat{k} \in \mathbb{N}$ such that for every $k \geq \hat{k}$ the element $g_{1}=a^{k} t a^{k} t^{-1}$ is commensurable with $g_{2}=a^{k} u a^{k} u^{-1}$ in $G$. Then there are $\beta, \gamma \in H_{\lambda_{0}}$ and $\epsilon, \xi \in\{-1,1\}$ such that $u=\gamma t^{\xi} \beta, \beta a \beta^{-1}=a^{\epsilon}, \gamma^{-1} a \gamma=a^{\epsilon}$.

Proof. Changing the finite relative generating set $\mathcal{X}$ of $G$, if necessary, we can assume that $t, u, t^{-1}, u^{-1} \in \mathcal{X}$. Let the finite subset $\Omega \subset G$ and the constant $K \in \mathbb{N}$ be 
chosen according to Lemma 6.1. Define $m=7 \mathrm{~K}$ and suppose that $k$ is large enough to satisfy $a^{k} \notin\left\{\left.h \in\langle\Omega\rangle|| h\right|_{\Omega} \leq m\right\}$.

Since $g_{1}$ and $g_{2}$ are commensurable, there exist $l, l^{\prime} \in \mathbb{Z} \backslash\{0\}$ and $y \in G$ such that $y g_{2}^{l} y^{-1}=g_{1}^{l^{\prime}}$. Let $C=|y|_{x \cup \mathscr{H}}, d=8$ and $L=L(C, d)$ be the constant from Lemma 6.5. Without loss of generality, assume that $4 l \geq L$. Consider the cycle $o=r q r^{\prime} q^{\prime}$ in $\Gamma(G, \mathcal{X} \cup \mathscr{H})$ such that $r$ and $r^{\prime}$ are paths of length $C$ whose labels represent $y$ in $G, r_{-}=1, q_{-}=r_{+}=y, \operatorname{Lab}(q) \equiv\left(a^{k} u a^{k} u^{-1}\right)^{l} \in \mathcal{W}(\Omega, m)$, $\mathrm{L}(q)=4 l, r_{-}^{\prime}=q_{+}, q_{-}^{\prime}=r_{+}^{\prime}=y g_{2}^{l} y^{-1}, \operatorname{Lab}\left(q^{\prime}\right) \equiv\left(a^{k} t a^{k} t^{-1}\right)^{l^{\prime}} \in \mathcal{W}(\Omega, m)$, $\mathrm{L}\left(q^{\prime}\right)=4 l^{\prime}$.

Now, by Lemma 6.5, there are subpaths $\tilde{q}=p_{1} s_{1} p_{2} s_{2} p_{3} s_{3} p_{4}$ of $q$ and $\tilde{q}^{\prime}=$ $p_{1}^{\prime} s_{1}^{\prime} p_{2}^{\prime} s_{2}^{\prime} p_{3}^{\prime} s_{3}^{\prime} p_{4}^{\prime}$ of $q^{\prime-1} \operatorname{such}$ that $\operatorname{Lab}\left(p_{i}\right) \equiv a^{k}, \operatorname{Lab}\left(p_{i}^{\prime}\right) \equiv a^{\epsilon k}, i=1,2,3,4$, for some $\epsilon \in\{-1,1\}$ (which depends on the sign of $\left.l^{\prime}\right), \operatorname{Lab}\left(s_{1}\right) \equiv \operatorname{Lab}\left(s_{3}\right) \equiv u$, $\operatorname{Lab}\left(s_{2}\right) \equiv u^{-1}, \operatorname{Lab}\left(s_{1}^{\prime}\right) \equiv \operatorname{Lab}\left(s_{3}^{\prime}\right) \equiv t^{\xi}, \operatorname{Lab}\left(s_{2}^{\prime}\right) \equiv t^{-\xi}$, for some $\xi \in\{-1,1\}$, and $p_{i}$ is connected in $\Gamma(G, \mathcal{X} \cup \mathscr{H})$ to $p_{i}^{\prime}$ for each $i=1,2,3,4$. Therefore there exist paths $\tilde{p}_{1}, \tilde{p}_{2}, \tilde{p}_{3}, \tilde{p}_{4}$ whose labels represent the elements $\alpha, \beta, \gamma, \delta \in H_{\lambda_{0}}$ respectively, such that $\left(\tilde{p}_{1}\right)_{-}=\left(p_{1}\right)_{+},\left(\tilde{p}_{1}\right)_{+}=\left(p_{1}^{\prime}\right)_{+},\left(\tilde{p}_{2}\right)_{-}=\left(p_{2}^{\prime}\right)_{+},\left(\tilde{p}_{2}\right)_{+}=\left(p_{2}\right)_{+}$, $\left(\tilde{p}_{3}\right)_{-}=\left(p_{3}\right)_{-},\left(\tilde{p}_{3}\right)_{+}=\left(p_{3}^{\prime}\right)_{-},\left(\tilde{p}_{4}\right)_{-}=\left(p_{4}^{\prime}\right)_{-},\left(\tilde{p}_{4}\right)_{+}=\left(p_{4}\right)_{-}($see Figure 2$)$.

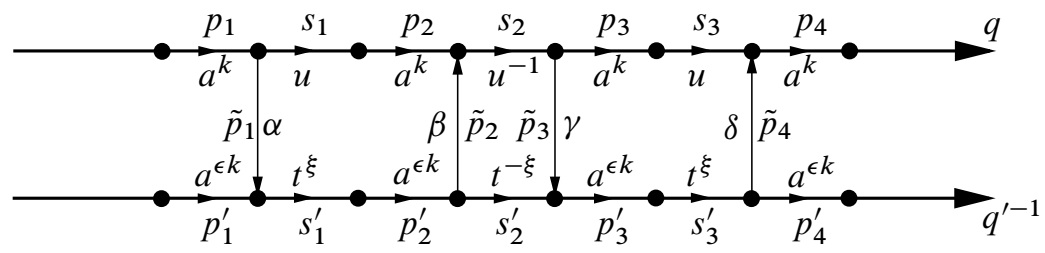

Figure 2.

The cycles $s_{1}^{-1} \tilde{p}_{1} s_{1}^{\prime} p_{2}^{\prime} \tilde{p}_{2} p_{2}^{-1}, s_{2} \tilde{p}_{3} s_{2}^{\prime-1} \tilde{p}_{2}$ and $s_{3}^{-1} p_{3}^{-1} \tilde{p}_{3} p_{3}^{\prime} s_{3}^{\prime} \tilde{p}_{4}$ give rise to the following equalities in the group $G$ :

$$
u=\alpha t^{\xi} a^{\epsilon k} \beta a^{-k}, u=\gamma t^{\xi} \beta \text { and } u=a^{-k} \gamma a^{\epsilon k} t^{\xi} \delta .
$$

Consequently, recalling that $H_{\lambda_{0}}$ is malnormal (Lemma 2.3) and that $t^{\xi} \notin H_{\lambda_{0}}$, we get

$$
\beta a^{k} \beta^{-1} a^{-\epsilon k}=t^{-\xi} \gamma^{-1} \alpha t^{\xi} \in H_{\lambda_{0}} \cap t^{-\xi} H_{\lambda_{0}} t^{\xi}=\{1\},
$$

and

$$
a^{-\epsilon k} \gamma^{-1} a^{k} \gamma=t^{\xi} \delta \beta^{-1} t^{-\xi} \in H_{\lambda_{0}} \cap t^{\xi} H_{\lambda_{0}} t^{-\xi}=\{1\}
$$

Thus

$$
\beta a^{k} \beta^{-1}=a^{\epsilon k} \quad \text { and } \quad \gamma^{-1} a^{k} \gamma=a^{\epsilon k}
$$


for some $\beta=\beta(k), \gamma=\gamma(k) \in H_{\lambda_{0}}$ and $\epsilon=\epsilon(k), \xi=\xi(k) \in\{-1,1\}$. Note that the proof works for any sufficiently large $k$, therefore we can find two mutually prime positive integers $k, k^{\prime}$ with the above properties such that $\epsilon(k)=\epsilon\left(k^{\prime}\right)=\epsilon$ and $\xi(k)=\xi\left(k^{\prime}\right)=\xi$. Denote $\beta^{\prime}=\beta\left(k^{\prime}\right)$ and $\gamma^{\prime}=\gamma\left(k^{\prime}\right)$, then $\gamma t^{\xi} \beta=u=\gamma^{\prime} t^{\xi} \beta^{\prime}$, implying

$$
\gamma^{-1} \gamma^{\prime}=t^{\xi} \beta \beta^{\prime-1} t^{-\xi} \in H_{\lambda_{0}} \cap t^{\xi} H_{\lambda_{0}} t^{-\xi}=\{1\} \text {. }
$$

Hence $\beta^{\prime}=\beta, \gamma^{\prime}=\gamma$,

$$
\beta a^{k^{\prime}} \beta^{-1}=a^{\epsilon k^{\prime}} \text { and } \gamma^{-1} a^{k^{\prime}} \gamma=a^{\epsilon k^{\prime}} .
$$

It remains to observe that since $k$ and $k^{\prime}$ are mutually prime, the formulas (6.1) and (6.2) together yield

$$
\beta a \beta^{-1}=a^{\epsilon} \quad \text { and } \quad \gamma^{-1} a \gamma=a^{\epsilon},
$$

\section{Small cancellation over relatively hyperbolic groups}

Let $G$ be a group generated by a subset $\mathcal{A} \subseteq G$ and let $\mathcal{O}$ be the set of all words in the alphabet $\mathcal{A}^{ \pm 1}$, that are trivial in $G$. Then $G$ has a presentation of the following form:

$$
G=\langle\mathcal{A} \mid \mathcal{O}\rangle .
$$

Given a symmetrized set of words $\mathcal{R}$ over the alphabet $\mathcal{A}$, consider the group $G_{1}$ defined by

$$
G_{1}=\langle\mathcal{A} \mid \mathcal{O} \cup \mathcal{R}\rangle=\langle G \mid \mathcal{R}\rangle .
$$

During the proof of the main result of this section we use presentations (7.2) (or, equivalently, the sets of additional relators $\mathcal{R}$ ) that satisfy the generalized small cancellation condition $C_{1}(\varepsilon, \mu, \lambda, c, \rho)$. In the case of word hyperbolic groups this condition was suggested by Ol'shanskii in [16], and was afterwards generalized to relatively hyperbolic groups by Osin in [21]. For the definition and detailed theory we refer the reader to the paper [21], as we will only use the properties, that were already established there. The following observation is an immediate consequence of the definition:

Remark 7.1. Let the constants $\varepsilon_{j}, \mu_{j}, \lambda, c, \rho_{j}, j=1,2$, satisfy $0<\lambda \leq 1,0 \leq$ $\varepsilon_{1} \leq \varepsilon_{2}, c \geq 0,0<\mu_{2} \leq \mu_{1}, \rho_{2} \geq \rho_{1}>0$. If the presentation (7.2) enjoys the condition $C_{1}\left(\varepsilon_{2}, \mu_{2}, \lambda, c, \rho_{2}\right)$ then it also enjoys the condition $C_{1}\left(\varepsilon_{1}, \mu_{1}, \lambda, c, \rho_{1}\right)$.

We will also assume that the reader is familiar with the notion of a van Kampen diagram over the group presentation (7.2) (see [10, Chapter V] or [15, Chapter 4]). Let $\Delta$ be such a diagram. A cell $\Pi$ of $\Delta$ is called an $\mathcal{R}$-cell if the label of its 
boundary contour $\partial \Pi$ (i.e., the word written on it starting with some vertex in the counter-clockwise direction) belongs to $\mathcal{R}$.

Consider a simple closed path $o=r q r^{\prime} q^{\prime}$ in a diagram $\Delta$ over the presentation (7.2), such that $q$ is a subpath of the boundary cycle of an $\mathcal{R}$-cell $\Pi$ and $q^{\prime}$ is a subpath of $\partial \Delta$. Let $\Gamma$ denote the subdiagram of $\Delta$ bounded by $o$. Assuming that $\Gamma$ has no holes, no $\mathcal{R}$-cells and $\mathrm{L}(r), \mathrm{L}\left(r^{\prime}\right) \leq \varepsilon$, it will be called an $\varepsilon$-contiguity subdiagram of $\Pi$ to $\partial \Delta$. The ratio $\mathrm{L}(q) / \mathrm{L}(\partial \Pi)$ will be called the contiguity degree of $\Pi$ to $\partial \Delta$ and denoted $(\Pi, \Gamma, \partial \Delta)$.

A diagram is said to be reduced if it has a minimal number of $\mathcal{R}$-cells among all the diagrams with the same boundary label.

If $G$ is a group hyperbolic relative to a family of proper subgroups $\left\{H_{i}\right\}_{i \in I}$, with a finite relative generating set $\mathcal{X}$, then $G$ is generated by the set $\mathcal{A}=\mathcal{X} \cup \bigcup_{i \in I}\left(H_{i} \backslash\right.$ $\{1\})$, and the Cayley graph $\Gamma(G, \mathcal{A})$ is a hyperbolic metric space [20, Corollary 2.54].

As for every condition of small cancellation, the main statement of the theory is the following analogue of Greendlinger's Lemma, claiming the existence of a cell, large part of whose contour lies on the boundary of the van Kampen diagram.

Lemma 7.2 ([21], Corollary 4.4). Suppose that the group $G$ is generated by a subset $\mathcal{A}$ such that the Cayley graph $\Gamma(G, \mathcal{A})$ is hyperbolic. Then for any $0<\lambda \leq 1$ there is $\mu_{0}>0$ such that for any $\mu \in\left(0, \mu_{0}\right]$ and $c \geq 0$ there are $\varepsilon_{0} \geq 0$ and $\rho_{0}>0$ with the following property.

Let the symmetrized presentation (7.2) satisfy the $C_{1}\left(\varepsilon_{0}, \mu, \lambda, c, \rho_{0}\right)$-condition. Further, let $\Delta$ be a reduced van Kampen diagram over $G_{1}$ whose boundary contour is $(\lambda, c)$-quasigeodesic in $G$. Then, provided $\Delta$ has an $\mathcal{R}$-cell, there exists an $\mathcal{R}$-cell $\Pi$ in $\Delta$ and an $\varepsilon_{0}$-contiguity subdiagram $\Gamma$ of $\Pi$ to $\partial \Delta$, such that

$$
(\Pi, \Gamma, \partial \Delta)>1-23 \mu \text {. }
$$

The main application of this particular small cancellation condition is

Lemma 7.3 ([21], Lemmas 5.1 and 6.3). For any $0<\lambda \leq 1, c \geq 0$ and $N>0$ there exist $\mu_{1}>0, \varepsilon_{1} \geq 0$ and $\rho_{1}>0$ such that for any symmetrized set of words $\mathcal{R}$ satisfying $C_{1}\left(\varepsilon_{1}, \mu_{1}, \lambda, c, \rho_{1}\right)$-condition the following hold.

1. The group $G_{1}$ defined by (7.2) is hyperbolic relative to the collection of images $\left\{\eta\left(H_{i}\right)\right\}_{i \in I}$ under the natural homomorphism $\eta: G \rightarrow G_{1}$.

2. The restriction of $\eta$ to the subset of elements having length at most $N$ with respect to $A$ is injective.

3. Any element that has a finite order in $G_{1}$ is an image of an element of finite order in $G$.

Below is the principal lemma of this section that will later be used to prove Theorem 1.5. 
Lemma 7.4. Assume that $G$ is a torsion-free group hyperbolic relative to a family of proper subgroups $\left\{H_{i}\right\}_{i \in I}, \mathcal{X}$ is a finite relative generating set of $G, S$ is a suitable subgroup of $G$ and $U \subset G$ is a finite subset. Suppose that $i_{0} \in I, a \in H_{i_{0}} \backslash\{1\}$ and $v_{1}, v_{2} \in G$ are hyperbolic elements which are not commensurable to each other. Then there exists a word $W(x, y)$ over the alphabet $\{x, y\}$ such that the following is true.

Denote $w_{1}=W\left(a, v_{1}\right) \in G, w_{2}=W\left(a, v_{2}\right) \in G$, and let $\left\langle\left\langle w_{2}\right\rangle\right\rangle$ be the normal closure of $w_{2}$ in $G, G_{1}=G /\left\langle\left\langle w_{2}\right\rangle\right\rangle$ and $\eta: G \rightarrow G_{1}$ be the natural epimorphism. Then

- $\eta$ is injective on $\left\{H_{\lambda}\right\}_{\lambda \in \Lambda} \cup U$ and $G_{1}$ is hyperbolic relative to the family $\left\{\eta\left(H_{\lambda}\right)\right\}_{\lambda \in \Lambda}$

- $\eta(S)$ is a suitable subgroup of $G_{1}$;

- $G_{1}$ is torsion-free;

- $\eta\left(w_{1}\right) \neq 1$.

Proof. By Lemma 2.7 there are hyperbolic elements $v_{3}, v_{4} \in S$ such that $v_{i} \not \approx v_{j}$ if $1 \leq i<j \leq 4$. Then by Lemma 2.2, the group $G$ is hyperbolic relative to the finite collection of subgroups $\left\{H_{i}\right\}_{i \in I} \cup \bigcup_{j=1}^{4}\left\{E_{G}\left(v_{j}\right)\right\}$, and generated by the set

$$
\mathcal{A}=\mathcal{X} \cup\left(\bigcup_{i \in I} H_{i} \cup \bigcup_{j=1}^{4} E_{G}\left(v_{j}\right)\right) \backslash\{1\} .
$$

Let $\Omega \subset G$ and $K \in \mathbb{N}$ denote the finite subset and the constant achieved after an application of Lemma 6.1 to this new collection of peripheral subgroups.

Define $m=7 K, \lambda=1 / 3, c=2$ and $N=\max \left\{|u|_{\mathcal{A}} \mid u \in U\right\}+1$. Choose $\mu_{j}>0, \varepsilon_{j} \geq 0$ and $\rho_{j}>0, j=0,1$, according to the claims of Lemmas 7.2 and 7.3. Let $\varepsilon=\max \left\{\varepsilon_{0}, \varepsilon_{1}\right\}$, and let $L=L(C, d)>0$ be the constant given by Lemma 6.5 where $C=\varepsilon_{0}$ and $d=2$. Evidently there exists $n \in \mathbb{N}$ such that, for $\mu=(3 \varepsilon+11) / n$, one has

$$
0<\mu \leq \min \left\{\mu_{0}, \mu_{1}\right\}, 2 n(1-23 \mu)>L, \text { and } 2 n>\max \left\{\rho_{0}, \rho_{1}\right\} .
$$

Set

$$
\mathscr{F}(\varepsilon)=\{h \in\langle\Omega\rangle|| h \mid \leq \max \{K(32 \varepsilon+70), m\}\} .
$$

Since the subset $\mathcal{F}(\varepsilon)$ is finite, we can find $k \in \mathbb{N}$ such that $a^{k^{\prime}}, v_{1}^{k^{\prime}}, v_{2}^{k^{\prime}} \notin \mathcal{F}(\varepsilon)$ whenever $k^{\prime} \geq k$. Consider the word

$$
W(x, y) \equiv x^{k} y^{k} x^{k+1} y^{k+1} \ldots x^{k+n-1} y^{k+n-1} .
$$

Let $w_{j} \in G$ be the element represented by the word $W\left(a, v_{j}\right)$ in $G, j=1,2$, and let $\mathcal{R}$ be the set of all cyclic shifts of $W\left(a, v_{2}\right)$ and their inverses. By Lemma 2.3, 
$H_{i_{0}} \cap E_{G}\left(v_{2}\right)=\{1\}$ because $G$ is torsion-free, hence by [21, Theorem 7.5] the presentation (7.2) satisfies the condition $C_{1}(\varepsilon, \mu, 1 / 3,2,2 n)$, and therefore, by Remark 7.1, it satisfies the conditions $C_{1}\left(\varepsilon_{0}, \mu, 1 / 3,2, \rho_{0}\right)$ and $C_{1}\left(\varepsilon_{1}, \mu_{1}, 1 / 3,2, \rho_{1}\right)$.

Observe that $w_{1} \neq 1$ in $G$ because, otherwise, there would have existed a closed path $q$ in $\Gamma(G, \mathcal{A})$ labelled by the word $W\left(a, v_{1}\right)$, and, by part (a) of Lemma 6.3, all components of $q$ would have been regular in the cycle $o=r q r^{\prime} q^{\prime}$ (where $r, r^{\prime}, q^{\prime}$ are trivial paths), which is obviously impossible.

Denote $G_{1}=G /\left\langle\left\langle w_{2}\right\rangle\right\rangle$ and let $\eta: G \rightarrow G_{1}$ be the natural epimorphism. Then, according to Lemma 7.3, the group $G_{1}$ is torsion-free, hyperbolic relative to

$$
\left\{\eta\left(H_{i}\right)\right\}_{i \in I} \cup \bigcup_{j=1}^{4}\left\{\eta\left(E_{G}\left(v_{j}\right)\right)\right\}
$$

and $\eta$ is injective on the set

$$
\bigcup_{i \in I} H_{i} \cup \bigcup_{j=1}^{4} E_{G}\left(v_{j}\right) \cup U
$$

(because the length in $\mathcal{A}$ of any element from this set is at most $N$ ). Since any elementary group is word hyperbolic, $G_{1}$ is also hyperbolic relative to $\left\{\eta\left(H_{i}\right)\right\}_{i \in I}$ (by Lemma 2.4) and $\eta\left(v_{3}\right), \eta\left(v_{4}\right) \in \eta(S)$ become hyperbolic elements of infinite order in $G_{1}$, that are not commensurable with each other (by Lemma 2.3). Therefore $E_{G_{1}}\left(\eta\left(v_{3}\right)\right) \cap E_{G_{2}}\left(\eta\left(v_{4}\right)\right)=\{1\}$ (recall that these subgroups are cyclic by Lemma 2.2 and because $G_{1}$ is torsion-free), and, consequently, $\eta(S)$ is a suitable subgroup of $G_{1}$.

Suppose that $\eta\left(w_{1}\right)=1$. By van Kampen's Lemma there exists a reduced planar diagram $\Delta$ over the presentation (7.2) with the word $W\left(a, v_{1}\right)$ written on its boundary. Since $W\left(a, v_{1}\right) \neq 1, \Delta$ possesses at least one $\mathcal{R}$-cell. It was proved in [21, Lemma 7.1] that any path in $\Gamma(G, \mathcal{A})$ labelled by $W\left(a, v_{1}\right)$ is $(1 / 3,2)$-quasigeodesic, hence we can apply Lemma 7.2 to find an $\mathcal{R}$-cell $\Pi$ of $\Delta$ and an $\varepsilon_{0}$-contiguity subdiagram $\Gamma$ (containing no $\mathcal{R}$-cells) between $\Pi$ and $\partial \Delta$ such that $(\Pi, \Gamma, \partial \Delta)>$ $1-23 \mu$. Thus there exists a cycle $o=r q r^{\prime} q^{\prime}$ in $\Gamma(G, \mathcal{A})$ such that $q$ is labelled by a subword of (a cyclic shift of) $W\left(a, v_{2}\right), q^{\prime}$ is labelled by a subword of (a cyclic shift of) $W\left(a, v_{1}\right)^{ \pm 1}, \mathrm{~L}(r), \mathrm{L}\left(r^{\prime}\right) \leq \varepsilon_{0}=C$ and

$$
\mathrm{L}(q)>(1-23 \mu) \cdot \mathrm{L}(\partial \Pi)=(1-23 \mu) \cdot 2 n>L .
$$

In particular, $\operatorname{Lab}(q), \operatorname{Lab}\left(q^{\prime}\right) \in \mathcal{W}(\Omega, m)$. Therefore we can apply Lemma 6.5 to find two consecutive components of $q$ that are connected to some components of $q^{\prime}$. Due to the form of the word $W\left(a, v_{2}\right)$, one of the formers will have to be an $E_{G}\left(v_{2}\right)$-component, but $q^{\prime}$ can have only $E_{G}\left(v_{1}\right)$ - or $H_{i_{0}}$-components. This yields a contradiction because $E_{G}\left(v_{2}\right) \neq E_{G}\left(v_{1}\right)$ and $E_{G}\left(v_{2}\right) \neq H_{i_{0}}$. Hence $\eta\left(w_{1}\right) \neq 1$ in $G_{1}$, and the proof is complete. 


\section{Every group is a group of outer automorphisms of a (2CC)-group}

Lemma 8.1. There exists a word $R(x, y)$ over the two-letter alphabet $\{x, y\}$ such that every non-elementary torsion-free word hyperbolic group $F_{1}$ has a non-elementary torsion-free word hyperbolic quotient $F$ that is generated by two elements $a, b \in F$ satisfying

$$
R(a, b) \stackrel{F}{\neq} 1, R\left(a^{-1}, b^{-1}\right) \stackrel{F}{=} 1, R(b, a) \stackrel{F}{=} 1, R\left(b^{-1}, a^{-1}\right) \stackrel{F}{=} 1 .
$$

Proof. Consider the word

$$
R(x, y) \equiv x y^{101} x^{2} y^{102} \ldots x^{100} y^{200} .
$$

Denote by $F(a, b)$ the free group with the free generators $a, b$. Let

$$
\mathcal{R}_{1}=\left\{R(a, b), R\left(a^{-1}, b^{-1}\right), R(b, a), R\left(b^{-1}, a^{-1}\right)\right\},
$$

and $\mathcal{R}_{2}$ be the set of all cyclic permutations of words from $\mathcal{R}_{1}^{ \pm 1}$. It is easy to see that the set $\mathcal{R}_{2}$ satisfies the classical small cancellation condition $C^{\prime}(1 / 8)$ (see [10, Chapter V]). Denote by $\tilde{N}$ the normal closure of the set

$$
\mathcal{R}_{3}=\left\{R\left(a^{-1}, b^{-1}\right), R(b, a), R\left(b^{-1}, a^{-1}\right)\right\}
$$

in $F(a, b)$. Since the symmetrization of $\mathcal{R}_{3}$ also satisfies $C^{\prime}(1 / 8)$, the group $\tilde{F}=$ $F(a, b) / \tilde{N}$ is a torsion-free ([10, Theorem V.10.1]) word hyperbolic group (because it has a finite presentation for which the Dehn function is linear by [10, Theorem V.4.4]) such that

$$
R(a, b) \stackrel{\tilde{F}}{\neq} 1 \text { but } R\left(a^{-1}, b^{-1}\right) \stackrel{\tilde{F}}{=} R(b, a) \stackrel{\tilde{F}}{=} R\left(b^{-1}, a^{-1}\right) \stackrel{\tilde{F}}{=} 1 .
$$

Indeed, if the word $R(a, b)$ were trivial in $\tilde{F}$ then, by Greendlinger's Lemma [10, Theorem V.4.4], it would contain more than a half of a relator from (the symmetrization of) $\mathcal{R}_{3}$ as a subword, which would contradict the fact that $\mathcal{R}_{2}$ enjoys $C^{\prime}(1 / 8)$. The group $\tilde{F}$ is non-elementary because every torsion-free elementary group is cyclic, hence, abelian, but in any abelian group the relation $R\left(a^{-1}, b^{-1}\right)=1$ implies $R(a, b)=1$.

Now, the free product $\tilde{G}=\tilde{F} * F_{1}$ is a torsion-free hyperbolic group. Its subgroups $\tilde{F}$ and $F_{1}$ are non-elementary, hence, according to a theorem of Ol'shanskii [16, Theorem 2], there exists a non-elementary torsion-free word hyperbolic group $F$ and a homomorphism $\phi: \tilde{G} \rightarrow F$ such that $\phi(\tilde{F})=\phi\left(F_{1}\right)=F$ and $\phi(R(a, b)) \neq 1$ in $F$. Therefore $F$ is a quotient of $F_{1}$, the ( $\phi$-images of the) elements $a, b$ generate $F$ and enjoy the required relations.

We are now ready to prove Theorem 1.5. 
Proof of Theorem 1.5. The argument will be similar to the one used to prove Theorem 5.1.

First, set $n=2$ and apply Lemma 4.2 to find a countable torsion-free group $H$ and a normal subgroup $M \triangleleft H$, where $H / M \cong C$ and $M$ has (2CC) (alternatively, one could start with a free group $H^{\prime}$ and $M^{\prime} \triangleleft H^{\prime}$ such that $H^{\prime} / M^{\prime} \cong C$, and then apply Lemma 4.4 to the pair $\left(H^{\prime}, M^{\prime}\right)$ to obtain $H$ and $M$ with these properties). Consider the word $R(x, y)$ and the torsion-free hyperbolic group $F$, generated by the elements $a, b \in F$ which satisfy (8.1), given by Lemma 8.1. Denote $G(-2)=H * F$ and let $N(-2)$ be the normal closure of $\langle M, F\rangle$ in $G(-2), F(-2)=F, \mathfrak{R}(-2)=\{R(a, b)\}$ - a finite subset of $F(-2)$. By Lemma 2.6, $G(-2)$ will be hyperbolic relative to the subgroup $H, G(-2)=H \cdot N(-2), H \cap N(-2)=M$ and $F(-2)$ will be a suitable subgroup of $G(-2)$.

The element $a \in F(-2)$ will be hyperbolic in $G(-2)$ and since the group $G(-2)$ is torsion-free, the maximal elementary subgroup $E_{G(-2)}(a)$ will be cyclic generated by some element $h_{-2} x_{-2}$, where $h_{-2} \in H, x_{-2} \in N(-2)$.

Choose $y_{-2} \in M$ so that $h_{-2} y_{-2} \neq 1$. By Lemmas 2.2 and 2.5, the HNN-extension

$$
G(-3 / 2)=\left\langle G(-2), t_{-1} \mid t_{-1} h_{-2} x_{-2} t_{-1}^{-1}=h_{-2} y_{-2}\right\rangle
$$

is hyperbolic relative to $H$. As in proof of Theorem 5.1, one can verify that $F(-3)$ is a suitable subgroup of $G(-3 / 2)$, and apply Theorem 2.8 to find an epimorphism $\eta_{-2}: G(-3 / 2) \rightarrow G(-1)$ such that $G(-1)$ is a torsion-free group hyperbolic relative to $\eta_{-2}(H), \eta_{-2}$ is injective on $H \cup \Re(-2)$ and $\eta_{-2}\left(t_{-1}\right) \in F(-1)$ where $F(-1)=$ $\eta_{-2}(F(-2))$ is a suitable subgroup of $G(-1)$. Hence $\eta_{-2}(G(-2))=G(-1)$ as $G(-3 / 2)$ was generated by $G(-2)$ and $t_{-1}$.

Denote $N(-1)=\eta_{-2}(N(-2)), \Re(-1)=\eta_{-2}(\Re(-2))$ and $\psi_{-2}=\left.\eta_{-2}\right|_{G(-2)}:$ $G(-2) \rightarrow G(-1)$. One can show that $G(-1)=H \cdot N(-1)$ and $H \cap N(-1)=$ $M$ using the same arguments as in the proof of Theorem 5.1. According to the construction, we have

$$
\eta_{-2}\left(t_{-1}\right) \eta_{-2}(a) \eta_{-2}\left(t_{-1}^{-1}\right)=\eta\left(t_{-1} a t_{-1}^{-1}\right) \in N(-1) \cap H=M
$$

in $G(-1)$, therefore, since the conjugation by $\eta_{-2}\left(t_{-1}\right)$ is an inner automorphism of $F(-1)$, we can assume that $F(-1)$ is generated by $a_{-1}$ and $b_{-1}$, where $a_{-1} \in M$ and $R\left(a_{-1}, b_{-1}\right) \neq 1$ in $F(-1)$ (because $\eta_{-2}(R(a, b)) \neq 1$ in $F(-1)$ ).

Now, if $b_{-1}$ is not a hyperbolic element of $G(-1)$, i.e., if $b_{-1} \stackrel{G(-1)}{\sim} c$ for some $c \in H$, then $c \in N(-1) \cap H=M$, and since $M$ has (2CC) we can find $s_{-1} \in G(-1)$ such that $b_{-1}=s_{-1} a_{-1} s_{-1}^{-1}$. In this case we define $G(0)=G(-1), N(0)=N(-1)$, $F(0)=F(-1), \Re(0)=\Re(-1), a_{0}=a_{-1}, s_{0}=s_{-1}$ and $\psi_{-1}=i d_{G(-1)}$.

Otherwise, if $b_{-1}$ is hyperbolic in $G(-1)$, then we construct the group $G(0)$, and an epimorphism $\psi_{-1}: G(-1) \rightarrow G(0)$ in an analogous way, to make sure that $\eta_{-1}$ is injective on $H \cup \Re(-1), G(0)$ torsion-free and hyperbolic relative to (the image 
of) $H, F(0)=\psi_{-1}(F(-1))$ is a suitable subgroup of $G(0), G(0)=H \cdot N(0)$ and $H \cap N(0)=M$ where $N(0)=\psi_{-1}(N(-1))$, and $b_{0}=s_{0} a_{0} s_{0}^{-1}$ in $G(0)$ where $b_{0}=\psi_{-1}\left(b_{-1}\right), a_{0}=\psi_{-1}\left(a_{-1}\right)$ for some $s_{0} \in G(0)$

Enumerate all elements of $N(0):\left\{g_{0}, g_{1}, g_{2}, \ldots\right\}$, and of $G(0):\left\{q_{0}, q_{1}, q_{2}, \ldots\right\}$, so that $g_{0}=q_{0}=1$.

The groups $G(j)$ together with $N(j) \triangleleft G(j), F(j) \leq G(j)$, finite subsets $\Re(j) \subset G(j)$, and elements $a_{j}, s_{j} \in G(j), j=1,2, \ldots$, that we will construct shall satisfy the following properties:

$1^{\circ}$. for each $j \in \mathbb{N}$ there is an epimorphism $\psi_{j-1}: G(j-1) \rightarrow G(j)$ which is injective on $H \cup \Re(j-1) . F(j)=\psi_{j-1}(F(j-1)), N(j)=\psi_{j-1}(N(j-1))$, $a_{j}=\psi_{j-1}\left(a_{j-1}\right) \in M, s_{j}=\psi_{j-1}\left(s_{j-1}\right) \in G(j)$;

$2^{\circ}$. $G(j)$ is torsion-free and hyperbolic relative to (the image of) $H$, and $F(j) \leq$ $G(j)$ is a suitable subgroup generated by $a_{j}$ and $s_{j} a_{j} s_{j}^{-1}$;

$3^{\circ} . G(j)=H \cdot N(j), N(j) \triangleleft G(j)$ and $H \cap N(j)=M$;

$4^{\circ}$. the natural image $\bar{g}_{j}$ of $g_{j}$ in $G(j)$ belongs to $F(j)$;

$5^{\circ}$. there exists $z_{j} \in H$ such that $\bar{q}_{j} \stackrel{G(j)}{\sim} z_{j}$, where $\bar{q}_{j}$ is the image of $q_{j}$ in $G(j)$;

$6^{\circ}$. if $j \geq 1, \bar{q}_{j-1} \in G(j-1) \backslash H$ and for each $\hat{k} \in \mathbb{N}$ there is $k \geq \hat{k}$ such that $a_{j-1}^{k} s_{j-1} a_{j-1}^{k} s_{j-1}^{-1} \stackrel{G(j-1)}{\not} a_{j-1}^{k} \bar{q}_{j-1} a_{j-1}^{k} \bar{q}_{j-1}^{-1}$, then where $w_{j-1}=$ $R_{j-1}\left(a_{j-1}, s_{j-1} a_{j-1} s_{j-1}^{-1}\right)$ in $G(j-1)$, for some there is a word $R_{j-1}(x, y)$ over the two-letter alphabet $\{x, y\}$ which satisfies

$$
\begin{gathered}
\Re(j) \ni \psi_{j-1}\left(R_{j-1}\left(a_{j-1}, s_{j-1} a_{j-1} s_{j-1}^{-1}\right)\right) \neq 1 \text { and } \\
\psi_{j-1}\left(R_{j-1}\left(a_{j-1}, \bar{q}_{j-1} a_{j-1} \bar{q}_{j-1}^{-1}\right)\right)=1 \text { in } G(j) .
\end{gathered}
$$

Suppose that the groups $G(0), \ldots, G(i)$ have already been defined. The group $G(i+1)$ will be constructed in three steps.

First, assume that $\bar{q}_{i} \in G(i) \backslash H$ and for each $\hat{k} \in \mathbb{N}$ there is $k \geq \hat{k}$ such that $a_{i}^{k} s_{i} a_{i}^{k} s_{i}^{-1} \stackrel{G(i)}{\not} a_{i}^{k} \bar{q}_{i} a_{i}^{k} \bar{q}_{i}^{-1}$. Observe that $s_{i} \notin H$ because, otherwise, one would have $F(i) \subset H$, which is impossible as $F(i)$ is suitable in $G(i)$. Therefore, by Corollary 6.6, we can suppose that $k$ is so large that the elements $v_{1}=a_{i}^{k} s_{i} a_{i}^{k} s_{i}^{-1}$ and $v_{2}=a_{i}^{k} \bar{q}_{i} a_{i}^{k} \bar{q}_{i}^{-1}$ are hyperbolic in $G(i)$. Applying Lemma 7.4 we can find a word $W(x, y)$ over $\{x, y\}$ such that the group $G(i+1 / 3)=G(i) /\left\langle\left\langle W\left(a_{i}, v_{2}\right)\right\rangle\right\rangle$ and the natural epimorphism $\eta: G(i) \rightarrow G(i+1 / 3)$ satisfy the following: $\eta$ is injective on $H \cup \Re(i), G(i+1 / 3)$ is torsion-free and hyperbolic relative to the image of) $H, \eta(F(i)) \leq G(i+1 / 3)$ is a suitable subgroup, and $\eta\left(W\left(a_{i}, v_{1}\right)\right) \neq 1$. 
Define the word $R_{i}(x, y) \equiv W\left(x, x^{k} y^{k}\right)$. Then $R_{i}\left(a_{i}, s_{i} a_{i} s_{i}^{-1}\right)=W\left(a_{i}, v_{1}\right)$, $R_{i}\left(a_{i}, \bar{q}_{i} a_{i} \bar{q}_{i}^{-1}\right)=W\left(a_{i}, v_{2}\right)$ in $G(i)$, hence

$$
\eta\left(R_{i}\left(a_{i}, s_{i} a_{i} s_{i}^{-1}\right)\right) \neq 1 \text { and } \eta\left(R_{i}\left(a_{i}, \bar{q}_{i} a_{i} \bar{q}_{i}^{-1}\right)\right)=1 \text { in } G(i+1 / 3) .
$$

If, on the other hand, $\bar{q}_{i} \in H$ or there is $\hat{k} \in \mathbb{N}$ such that for every $k \geq \hat{k}$ one has $a_{i}^{k} s_{i} a_{i}^{k} s_{i}^{-1} \stackrel{G(i)}{\approx} a_{i}^{k} \bar{q}_{i} a_{i}^{k} \bar{q}_{i}^{-1}$, then we define $G(i+1 / 3)=G(i), \eta: G(i) \rightarrow$ $G(i+1 / 3)$ to be the identical homomorphism and $R_{i}(x, y)$ to be the empty word.

Let $\hat{g}_{i+1}$ and $\hat{q}_{i+1}$ denote the images of $g_{i+1}$ and $q_{i+1}$ in $G(i+1 / 3), \hat{N}(i)=$ $\eta(N(i)), \hat{F}(i)=\eta(F(i))$ and $\hat{\Re}(i)=\eta\left(\Re(i) \cup\left\{R_{i}\left(a_{i}, s_{i} a_{i} s_{i}^{-1}\right)\right\}\right)$. Then, using $3^{\circ}$, we get $G(i+1 / 3)=H \cdot \widehat{N}(i)$ and $H \cap \widehat{N}(i)=M$ because $\operatorname{ker}(\eta) \leq N(i)$ (as $a_{i}, \bar{q}_{i} a_{i} \bar{q}_{i}^{-1} \in N(i)$ ).

Now we construct the group $G(i+2 / 3)$ in exactly the same way as the group $G(i+1 / 2)$ was constructed in during the proof of Theorem 5.1.

If for some $f \in G(i+1 / 3), f \hat{q}_{i+1} f^{-1}=z \in H$, then set $G(i+2 / 3)=G(i)$, $K_{i+1}=\hat{N}(i) \triangleleft G(i+2 / 3)$ and $t_{i+1}=1$.

Otherwise, $\hat{q}_{i+1}$ is a hyperbolic element of infinite order in $G(i+1 / 3)$. Since $G(i+1 / 3)$ is torsion-free, one has $E_{G(i+1 / 3)}\left(\hat{q}_{i+1}\right)=\langle h x\rangle$ for some $h \in H$ and $x \in \hat{N}(i)$, and there is $m \in \mathbb{Z}$ such that $\hat{q}_{i+1}=(h x)^{m}$. Now, by Lemma 2.2, $G(i+1 / 3)$ is hyperbolic relative to $\{H,\langle h x\rangle\}$. Choose $y \in M$ so that $h y \neq 1$ and let $G(i+2 / 3)$ be the following HNN-extension of $G(i+1 / 3)$ :

$$
G(i+2 / 3)=\left\langle G(i+1 / 3), t_{i+1} \mid t_{i+1}(h x) t_{i+1}^{-1}=h y\right\rangle .
$$

The group $G(i+2 / 3)$ is torsion-free and hyperbolic relative to $H$ by Lemma 2.5 . One can show that $\hat{F}(i)$ is a suitable subgroup of $G(i+2 / 3)$ in the same way as during the proof of Theorem 5.1. Lemma 4.3 assures that $H \cap K_{i+1}=M$ where $K_{i+1} \triangleleft G(i+2 / 3)$ is the normal closure of $\left\langle\hat{N}(i), t_{i+1}\right\rangle$ in $G(i+2 / 3)$. Finally, note that

$$
t_{i+1} \hat{q}_{i+1} t_{i+1}^{-1}=t_{i+1}(h x)^{m} t_{i+1}^{-1}=(h y)^{m}=z \in H \text { in } G(i+2 / 3) .
$$

Define $T_{i+1}=\left\{\hat{g}_{i+1}, t_{i+1}\right\} \subset K_{i+1}$. The group $G(i+1)$ is constructed from $G(i+2 / 3)$ as follows. Since $T_{i+1} \cdot \hat{F}(i) \subset K_{i+1} \triangleleft G(i+2 / 3)$, we can apply Theorem 2.8 to find a group $G(i+1)$ and an epimorphism $\varphi_{i}: G(i+2 / 3) \rightarrow G(i+1)$ such that $\varphi_{i}$ is injective on $H \cup \widehat{\Re}(i), G(i+1)$ is torsion-free and hyperbolic relative to (the image of) $H,\left\{\varphi_{i}\left(\hat{g}_{i+1}\right), \varphi_{i}\left(t_{i+1}\right)\right\} \subset \varphi_{i}(\hat{F}(i)), \varphi_{i}(\widehat{F}(i))$ is a suitable subgroup of $G(i+1)$, and $\operatorname{ker}\left(\varphi_{i}\right) \leq K_{i+1}$. Denote by $\psi_{i}: G(i) \rightarrow G(i+1)$ the composition $\varphi_{i} \circ \eta$. Then $\psi_{i}(G(i))=\varphi_{i}(G(i))=G(i+1)$ because $G(i+2 / 3)$ was generated by $G(i)$ and $t_{i+1}$, and according to the construction, $t_{i+1} \in \varphi_{i}(\hat{F}(i)) \leq \varphi_{i}(G(i))$. Now, after defining $F(i+1)=\psi_{i}(F(i)), N(i+1)=\psi_{i}(N(i)), \Re(i+1)=\varphi_{i}(\hat{\Re}(i))$, $\bar{g}_{i+1}=\varphi_{i}\left(\hat{g}_{i+1}\right) \in F(i+1)$ and $z_{i+1}=\varphi_{i}(z) \in H$, we see that the conditions 
$1^{\circ}-5^{\circ}$ hold in the case when $j=i+1$, as in the proof of Theorem 5.1. The last property $6^{\circ}$ follows from the way we constructed the group $G(i+1 / 3)$.

Let $Q=G(\infty)$ be the direct limit of the sequence $\left(G(i), \psi_{i}\right)$ as $i \rightarrow \infty$, and let $F(\infty)$ and $N=N(\infty)$ be the limits of the corresponding subgroups. Let $a_{\infty}, b_{\infty}$ and $s_{\infty}$ be the images of $a_{0}, b_{0}$ and $s_{0}$ in $Q$ respectively. Then $b_{\infty}=s_{\infty} a_{\infty} s_{\infty}^{-1}, Q$ is torsion-free by $2^{\circ}, N \triangleleft Q, Q=H \cdot N$ and $H \cap N=M$ by $3^{\circ}, N \leq F(\infty)$ by $4^{\circ}$. Hence $Q / N \cong H / M \cong C$.

Since $F(0) \leq N(0)$ we get $F(\infty) \leq N$. Thus $N=F(\infty)$ is a homomorphic image of $F(0)=F$, and, consequently, it is a quotient of $F_{1}$. By $5^{\circ}$, for any $q \in N$ there are $z \in H$ and $p \in Q$ such that $p q p^{-1}=z$. Consequently $z \in H \cap N=M$. Choose $x \in N$ and $h \in H$ so that $p=h x$. Since $M$ has (2CC) and $h^{-1} z h \in M$, there is $y \in M$ such that $y h^{-1} z h y^{-1}=z$, therefore $(y x) q(y x)^{-1}=z \in M$ and $y x \in M N=N$. Hence each element $q$ of $N$ will be conjugated (in $N$ ) to an element of $M$, and since $M$ has (2CC), therefore the group $N$ will also have (2CC).

The property that $C_{Q}(N)=\{1\}$ can be established in the same way as in Theorem 5.1. Therefore the natural homomorphism $Q \rightarrow \operatorname{Aut}(N)$ is injective. It remains to show that it is surjective, that is for every $\phi \in \operatorname{Aut}(N)$ there is $g \in Q$ such that $\phi(x)=g x g^{-1}$ for every $x \in N$. Since all non-trivial elements of $N$ are conjugated, after composing $\phi$ with an inner automorphism of $N$, we can assume that $\phi\left(a_{\infty}\right)=a_{\infty}$. On the other hand, there exist $q_{\infty} \in N$ and $i \in \mathbb{N}$ such that $\phi\left(b_{\infty}\right)=q_{\infty} a_{\infty} q_{\infty}^{-1}$ and $q_{\infty}$ is the image of $q_{i}$ in $Q$. Note that $s_{\infty} \notin H$ because $s_{i} \in G(i) \backslash H$ for every $i \in \mathbb{N}$. This implies that $H$ is a proper subgroup of $N$, thus $q_{\infty} \notin H$ since $N=F(\infty)=\left\langle a_{\infty}, q_{\infty} a_{\infty} q_{\infty}^{-1}\right\rangle \leq Q$, and $a_{\infty} \in H$. Hence $\bar{q}_{i} \in G(i) \backslash H$.

Now we have to consider two possibilities.

Case 1 . For each $\hat{k} \in \mathbb{N}$ there is $k \geq \hat{k}$ such that

$$
a_{i}^{k} s_{i} a_{i}^{k} s_{i}^{-1} \stackrel{G(i)}{\not} a_{i}^{k} \bar{q}_{i} a_{i}^{k} \bar{q}_{i}^{-1}
$$

Then there is a word $R_{i}(x, y)$ such that the property $6^{\circ}$ holds for $j=i+1$. And, since each $\psi_{j}$ is injective on $\{1\} \cup \Re_{j}$ (by $2^{\circ}$ ), we conclude that

$$
R_{i}\left(a_{\infty}, s_{\infty} a_{\infty} s_{\infty}^{-1}\right) \neq 1 \quad \text { and } \quad R_{i}\left(a_{\infty}, q_{\infty} a_{\infty} q_{\infty}^{-1}\right)=1 \text { in } Q
$$

which contradicts the injectivity of $\phi$. Hence Case 1 is impossible.

Case 2. The assumptions of Case 1 fail. Then we can use Lemma 6.7 to find $\beta, \gamma \in H$ and $\epsilon, \xi \in\{-1,1\}$ such that $\bar{q}_{i}=\gamma s_{i}^{\xi} \beta, \beta a_{i} \beta^{-1}=a_{i}^{\epsilon}$ and $\gamma^{-1} a_{i} \gamma=a_{i}^{\epsilon}$ in $G(i)$. Denote by $\gamma_{\infty}$ the image $\gamma$ in $Q$, and for any $y \in Q$ let $C_{y}$ be the automorphism of $N$ defined by $C_{y}(x)=y x y^{-1}$ for all $x \in N$.

If $\xi=-1$ then $\gamma_{\infty}^{-1} a_{\infty} \gamma_{\infty}=a_{\infty}^{\epsilon}$ and $\phi\left(b_{\infty}\right)=q_{\infty} a_{\infty} q_{\infty}^{-1}=\gamma_{\infty} s_{\infty}^{-1} a_{\infty}^{\epsilon} s_{\infty} \gamma_{\infty}^{-1}$, 
hence

$$
\operatorname{Aut}(N) \ni C_{s_{\infty} \gamma_{\infty}^{-1}} \circ \phi:\left\{\begin{array}{l}
a_{\infty} \mapsto s_{\infty} a_{\infty}^{\epsilon} s_{\infty}^{-1}=b_{\infty}^{\epsilon}, \\
b_{\infty}=s_{\infty} a_{\infty} s_{\infty}^{-1} \mapsto a_{\infty}^{\epsilon} .
\end{array}\right.
$$

But $N$ has no such automorphisms because $R\left(a_{\infty}, b_{\infty}\right) \neq 1$ and $R\left(b_{\infty}^{\epsilon}, a_{\infty}^{\epsilon}\right)=1$ in $N$ (since $N$ is a quotient of $F$ and $1 \neq R\left(a_{0}, b_{0}\right) \in \Re(0)$ in $G(0)$ ).

Therefore $\xi=1$. Similarly, $\epsilon=1$, as otherwise we would obtain a contradiction with the fact that $R\left(a_{\infty}^{-1}, b_{\infty}^{-1}\right)=1$ in $N$. Thus

$$
\operatorname{Aut}(N) \ni C_{\gamma_{\infty}^{-1}} \circ \phi:\left\{\begin{array}{l}
a_{\infty} \mapsto a_{\infty}, \\
b_{\infty}=s_{\infty} a_{\infty} s_{\infty}^{-1} \mapsto s_{\infty} a_{\infty} s_{\infty}^{-1}=b_{\infty} .
\end{array}\right.
$$

And since $a_{\infty}$ and $b_{\infty}$ generate $N$ we conclude that for all $x \in N, \phi(x)=g x g^{-1}$, where $g=\gamma_{\infty} \in Q$. Thus the natural homomorphism from $Q$ to $\operatorname{Aut}(N)$ is bijective, implying that $\operatorname{Out}(N)=\operatorname{Aut}(N) / \operatorname{Inn}(N) \cong Q / N \cong C$.

\section{References}

[1] G. Arzhantseva, A. Minasyan, D. Osin, The SQ-universality and residual properties of relatively hyperbolic groups. J. Algebra 315 (2007), 165-177. Zbl 1132.20022 MR 2344339

[2] I. Belegradek, D. Osin, Rips construction and Kazhdan property (T). Groups Geom. Dyn. 2 (2008), 1-12. Zbl 05252431 MR 2367206

[3] I. Bumagin, D. T. Wise, Every group is an outer automorphism group of a finitely generated group. J. Pure Appl. Algebra 200 (2005), 137-147. Zbl 1082.20021 MR 2142354

[4] R. Camm, Simple Free Products. J. London Math. Soc. 28 (1953), 66-76. Zbl 0050.02001 MR 0052420

[5] Y. de Cornulier, Finitely presentable, non-Hopfian groups with Kazhdan's Property and infinite outer automorphism group. Proc. Amer. Math. Soc. 135 (2007), 951-959. Zbl 05120305 MR 2262894

[6] P. de la Harpe, A. Valette, La propriété (T) de Kazhdan pour les groupes localement compacts (avec un appendice de Marc Burger). Astérisque 175 (1989). Zbl 0759.22001 MR 1023471

[7] M. Droste, M. Giraudet, R. Göbel, All groups are outer automorphism groups of simple groups. J. London Math. Soc. (2) 64 (2001), 565-575. Zbl 1015.20027 MR 1865550

[8] G. Higman, B. H. Neumann, H. Neumann, Embedding theorems for groups. J. London Math. Soc. 24 (1949), 247-254. Zbl 0034.30101 MR 0032641

[9] The Kourovka notebook. Unsolved problems in group theory, 16th augmented edition (V. D. Mazurov and E. I. Khukhro, eds.), Siberian branch of Russian Academy of Sciences, Institute of Mathematics, Novosibirsk 2006. Zbl 1084.20001 MR 2263886

[10] R. Lyndon and P. Schupp, Combinatorial Group Theory. Ergeb. Math. Grenzgeb. 89, Springer-Verlag, Berlin 1977. Zbl 0368.20023 MR 0577064 
[11] T. Matumoto, Any group is represented by an outer automorphism group. Hiroshima Math. J. 19 (1989), 209-219. Zbl 0727.20029 MR 1009671

[12] A. Muranov, Diagrams with selection and method for constructing boundedly generated and boundedly simple groups. Comm. Algebra 33 (2005), 1217-1258. Zbl 02175993 MR 2136699

[13] A. Muranov, Finitely generated infinite simple groups of infinite commutator width. Internat. J. Algebra Comput. 17 (2007), 607-659. Zbl 05172239 MR 2333374

[14] Y. Ollivier, D. T. Wise, Kazhdan groups with infinite outer automorphism group. Trans. Amer. Math. Soc. 359 (2007), 1959-1976. Zbl 05120622 MR 2276608

[15] A. Yu. Ol'shanskii, Geometry of defining relations in groups. Nauka, Moscow 1989 (in Russian); English transl. Math. Appl. (Soviet Series) 70, Kluwer Academic Publishers, Dordrecht 1991. Zbl 0732.20019 MR 1191619

[16] A. Yu. Ol'shanskii, On residualing homomorphisms and $G$-subgroups of hyperbolic groups. Internat. J. Algebra Comput. 3 (1993), 365-409. Zbl 0830.20053 MR 1250244

[17] D. V. Osin, Elementary subgroups of relatively hyperbolic groups and bounded generation, Internat. J. Algebra Comput. 16 (2006), 99-118. Zbl 1100.20033 MR 2217644

[18] D. V. Osin, Peripheral fillings of relatively hyperbolic groups. Invent. Math. 167 (2007), 295-326. Zbl 1116.20031 MR 2270456

[19] D. V. Osin, Relative Dehn functions of HNN-extensions and amalgamated products. In Topological and asymptotic aspects of group theory, Contemp. Math. 394, Amer. Math. Soc., Providence, RI, 2006, 209-220. Zbl 1111.20036 MR 2216718

[20] D. V. Osin, Relatively hyperbolic groups: intrinsic geometry, algebraic properties, and algorithmic problems. Mem. Amer. Math. Soc. 179 (2006), no. 843. Zbl 1093.20025 MR 2182268

[21] D. V. Osin, Small cancellations over relatively hyperbolic groups and embedding theorems, Annals of Math., to appear; arXiv: math.GR/0411039. Zbl MR

[22] F. Paulin, Outer automorphisms of hyperbolic groups and small actions on $\mathbb{R}$-trees. In Arboreal Group Theory (MSRI, Berkeley, 1988), R. C. Alperin ed., Math. Sci. Res. Inst. Publ. 19, Springer, New York 1991. Zbl 0804.57002 MR 1105339

Received April 5, 2007

Ashot Minasyan, School of Mathematics, University of Southampton, Highfield, Southampton, SO17 1BJ, United Kingdom

E-mail: aminasyan@gmail.com 